\title{
Dark matter electromagnetic dipoles: the WIMP expectation
}

\author{
Thomas Hambye ${ }^{a}$ and Xun-Jie $\mathbf{X} \mathbf{u}^{a, b}$ \\ ${ }^{a}$ Service de Physique Théorique, Université Libre de Bruxelles, \\ Boulevard du Triomphe, CP225, 1050 Brussels, Belgium \\ ${ }^{b}$ Institute of High Energy Physics, Chinese Academy of Sciences, \\ Beijing 100049, China \\ E-mail: thomas.hambye@ulb.be, xunjie.xu@gmail.com
}

ABSTRACT: We perform a systematic study of the electric and magnetic dipole moments of dark matter (DM) that are induced at the one-loop level when DM experiences fourfermion interactions with Standard Model (SM) charged fermions. Related to their loop nature these moments can largely depend on the UV completion at the origin of the fourfermion operators. We illustrate this property by considering explicitly two simple ways to generate these operators, from $t$ - or $s$-channel tree-level exchange. Fixing the strength of these interactions from the DM relic density constraint, we obtain in particular a magnetic moment that, depending on the interaction considered, lies typically between $10^{-20}$ to $10^{-23} \mathrm{ecm}$ or identically vanishes. These non-vanishing values induce, via photon exchange, DM-nucleus scattering cross sections that could be probed by current or near future direct detection experiments.

Keywords: Cosmology of Theories beyond the SM, Beyond Standard Model

ARXIV EPRINT: 2106.01403 


\section{Contents}

1 Introduction 1

2 Framework 3

2.1 Effective interactions of DM 3

2.2 DM relic abundance 4

3 Loop-induced electromagnetic interactions $\quad 6$

3.1 Loop integrals with contact interactions 8

3.2 Including $t$ - and $s$-channel energy dependence 8

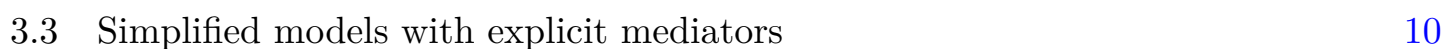

$\begin{array}{ll}3.4 & \text { UV complete example } \\ \end{array}$

$4 \quad$ Expected magnitude of electromagnetic dipoles $\quad 13$

5 Electromagnetic dipoles in direct detection $\quad 14$

6 Conclusion 20

$\begin{array}{ll}\text { A Loop calculations } & 21\end{array}$

\section{Introduction}

Weakly interacting massive particles (WIMPs) are among the best motivated dark matter (DM) candidates. As is well known, DM particles annihilating into lighter particles with coupling strength of order unity undergo a non-relativistic freeze-out in the primordial thermal bath of the Universe, leaving a relic density of the order of the observed one if the DM mass is roughly around the electroweak scale. This "WIMP miracle" has triggered vast experimental effort in DM searches — see [1-6] for reviews. In particular, over the past years, direct and indirect detection experiments have reached the sensitivity necessary to probe this paradigm in many different contexts. Collider experiments also offer possibilities of tests. A number of explicit models have been already excluded, whereas many other ones could be seriously tested in the near future.

In many models WIMPs annihilate into SM fermions via a $t$ - or $s$-channel mediator. If this mediator is sufficiently heavy, it can be integrated out, leading to a local effective interaction. Thus in this case the (tree level) phenomenology of the model reduces to the one that can be obtained from the effective field theory (EFT) for DM annihilation. As is well-known too, in this case one can get a one-to-one relation between the annihilation rate fixed by the relic density constraint and direct, indirect as well as collider signals. ${ }^{1}$

\footnotetext{
${ }^{1}$ Actually, the criteria of having a sufficiently heavy mediator for the EFT to be valid depends on the process considered, see e.g. [7-9].
} 
In this work we are interested in effective interactions involving charged SM fermions $(f)$ and DM fermions $(\chi)$, of the general form $\mathcal{L} \supset G \bar{\chi} \mathcal{O} \chi \bar{f} \mathcal{O}^{\prime} f$ with $\mathcal{O}$ and $\mathcal{O}^{\prime}$ any possible operators. From the relic density constraint, the dimensional coupling $G$ is typically of the order of $10^{-1} G_{F} \times\left(m_{\chi} / 100 \mathrm{GeV}\right)$ where $G_{F}$ is the Fermi constant and $m_{\chi}$ is the DM mass. If the SM fermion is a light quark and DM lies around the electroweak scale, such values of $G$ have already been ruled out by recent direct detection experiments for operators that lead to spin-independent (SI) cross sections on nuclei. These are in particular the XENON1T [10], LUX [11] and PandaX-II [12] experiments which have now put upper limits on the SI DM-nucleon cross section down to $\sim 10^{-46} \mathrm{~cm}^{2}$ for $m_{\chi}$ ranging from tens to hundreds of $\mathrm{GeV}$. However, WIMPs are not necessarily expected to dominantly couple to light quarks. For other SM fermions (e.g. $f=e, \mu, \tau, c, b, t, \nu_{e}, \nu_{\mu}, \nu_{\tau}$ ), direct detection bounds are generally weaker. In addition, various operators may lead to spin-dependent (SD) cross sections, for which the experimental sensitivity is weaker.

An interesting possibility to improve the direct detection sensitivity in such cases stems from the fact that WIMPs might have electromagnetic dipole moments. In fact, various electromagnetic form factors (electric/magnetic dipoles, anapole, charged radius) of WIMPs have been considered in the literature [13-33]. Early studies [17-20, 24] considered them as a solution to resolve the discrepancy between DAMA/CoGeNT signals and null results of other DM searches, though this has been since then well excluded. Collider, $\gamma$-ray, and CMB searches for dipole interacting DM have been studied in refs. [20, 21]. More recently, ref. [28] considered leptophilic DM and showed that its loop-induced electromagnetic dipoles led to restrictive direct detection bounds. ${ }^{2}$

In this work, instead of considering that the annihilation induced by the dipoles (into SM charged particles via photon exchange) is responsible for the relic density, as in many of these works (e.g. [15, 17-20]), or instead of assuming a specific model, we will start from the effective four-fermion operators, as ref. [16] considered. The question we ask is: once the coefficients of the effective operators are fixed by the relic density, what are the values of the dipoles one can expect at the one-loop level and what are their phenomenological consequences (mainly for direct detection)? The answer to this question is not straightforward because a procedure which would consist in simply computing the loop diagram resulting from closing the charged fermion line of the 4-fermion operator, and attaching an external photon to this fermion line, does not necessarily lead to the dipoles that would be generated in the UV complete theory that is at the origin of these operators. To understand better this issue we will consider a particularly simple example where the 4 -fermion operator is induced by the $t$-channel exchange of a heavy mediator. As we will show, for most operators the calculation of this contact interaction loop diagram (which corresponds to taking in the heavy mediator propagator only the first term in $q^{2} / M^{2}$ expansion, (i.e. $\left.1 /\left(q^{2}-M^{2}\right) \rightarrow-1 / M^{2}\right)$ leads to vanishing dipoles. However, the integration of the loop diagram with the full $t$-channel propagator (which corresponds to take higher order $q^{2} / M^{2}$ expansion terms) gives non-vanishing dipoles. In a full Effective Field Theory

\footnotetext{
${ }^{2}$ Beyond the WIMP regime, there has been growing interest in electromagnetic dipoles of sub-GeV DM due to potential connections with CMB/LSS observations, stellar physics, and the intensity frontier searches — see, e.g., [29-31, 33].
} 
this would correspond to taking also the loop contribution of higher dimensional operators. Moreover, as we will show too (considering explicit UV model examples), a UV complete theory is in general not expected to give only the 4-fermion interaction operator in the low energy limit. It may lead to other operators (in particular involving the photon field) whose loop contribution also gives dipole contributions (i.e. operators whose coefficients are not fixed by the relic density constraint and thus UV complete model dependent, that is to say unknown from the low energy point of view). In this work we will argue that despite of these issues, when we replace the contact interaction by the full heavy mediator propagator, the dipoles one gets are generic of what we can typically expect in UV complete models. To show that, one will also compute the dipoles considering explicit scalar or vector boson mediators (what we call "simplified models") and examples of UV complete models, and compare the results.

We then study the implication of non-vanishing dipole for direct detection. We find that the magnitude of the non-vanishing loop-induced dipoles, typically of the order of $10^{-20} \mathrm{ecm}$ ( or $10^{-20} m_{f} / m_{\chi}$ ecm), implies that DM-nucleus scattering via dipole interactions could be probed within current and future experimental sensitivities. In particular for operators involving heavy quarks or charged leptons, or when the DM-nucleus cross section is SD at tree level, this might provide the best possibility of probing these interactions and thus possibly the origin of the DM relic density. This stems from the fact that for low nuclear recoil energies the cross section is considerably enhanced by the exchange of a massless (photon) mediator.

The paper is organized as follows. In section 2, we present a complete description of the most general four-fermion interactions of DM fermions with SM fermions, and determine the interaction strength required to produce the observed relic abundance. In section 3 , we compute the loop-induced electromagnetic dipoles of DM by closing charged fermion loops, which we do, as said above, at various levels, including calculations for contact interactions, $s$ - and $t$-channel energy-dependent effective interactions, explicit scalar or vector boson mediators, and UV complete examples. The comparison of these various levels is quite interesting in our opinion. In section 4 we briefly summarize the typical magnitude of the dipoles one can expect when the four-fermion interaction strength is determined by the relic abundance. In section 5, the resulting magnitude of electromagnetic dipoles is confronted with direct detection limits obtained by investigating the recoil spectra of dipole-interacting DM. We conclude in section 6 and delegate the loop calculation details to the appendix.

\section{Framework}

\subsection{Effective interactions of DM}

We start with the most general four-fermion interactions of Dirac DM $(\chi)$ and SM fermions (f) $[16,34-38]$ :

$$
\mathcal{L} \supset G_{F} \sum_{a} \bar{\chi} \Gamma^{a} \chi \bar{f} \Gamma^{a}\left(\epsilon_{a}+\tilde{\epsilon}_{a} i_{a} \gamma^{5}\right) f,
$$

where the $\Gamma^{a}$ matrices (with $a=S, P, V, A, T$ ) span all the 16 possible independent combinations of Dirac matrices:

$$
\Gamma^{S}=I, \Gamma^{P}=i \gamma^{5}, \Gamma^{V}=\gamma^{\mu}, \Gamma^{A}=\gamma^{\mu} \gamma^{5}, \Gamma^{T}=\sigma^{\mu \nu} .
$$


We refer to the above five possible bi-linear products of Dirac spinors as scalar, pseudoscalar, vector, axial-vector, and tensor interactions. In eq. (2.1), we have normalized the interaction strength by the Fermi constant $G_{F}$ since in the WIMP paradigm, the interactions are typically of this magnitude. Potential deviations are absorbed into the dimensionless constants $\epsilon_{a}$ and $\tilde{\epsilon}_{a}$. Note that in eq. (2.1) we have inserted an $i_{a}$ factor, which is defined as $i_{S, P, T}=i$ and $i_{V, A}=1$, so that the various terms are hermitian, with $\epsilon_{a}$ and $\tilde{\epsilon}_{a}$ real numbers - for further discussions see e.g. refs. [39, 40]. For tensor interactions, one could consider adding $\gamma^{5}$ between $\bar{\chi}$ and $\chi$ but the operator $\bar{\chi} \sigma^{\mu \nu} \gamma^{5} \chi \bar{f} \sigma_{\mu \nu} f$ is actually identical $^{3}$ to $\bar{\chi} \sigma^{\mu \nu} \chi \bar{f} \sigma_{\mu \nu} \gamma^{5} f$. Hence eq. (2.1) provides a complete description of all possible Lorentz-invariant four-fermion interactions. This set of effective operators has also been frequently used for DM collider searches and direct detection — see e.g. refs. [16, 34-38]. Note importantly that the $S, P$ and $T$ operators are not SM gauge invariant, but could be generated through electroweak symmetry breaking, see the discussion in section 3.4.

In the SM fermion chiral basis, one can also write eq. (2.1) as

$$
\begin{aligned}
\mathcal{L} \supset & G_{F}\left[\epsilon_{S}^{L} \bar{\chi} \chi \overline{f_{R}} f_{L}+\epsilon_{P}^{L} \bar{\chi} i \gamma^{5} \chi \overline{f_{R}} f_{L}\right. \\
& +\epsilon_{V}^{L} \bar{\chi} \gamma^{\mu} \chi \overline{f_{L}} \gamma_{\mu} f_{L}+\epsilon_{A}^{L} \bar{\chi} \gamma^{\mu} \gamma^{5} \chi \overline{f_{L}} \gamma_{\mu} f_{L} \\
& \left.+\epsilon_{T}^{L} \bar{\chi} \sigma^{\mu \nu} \chi \overline{f_{R}} \sigma_{\mu \nu} f_{L}+(L \leftrightarrow R)\right],
\end{aligned}
$$

where $f_{L, R} \equiv P_{L, R} f, P_{L, R} \equiv\left(1 \mp \gamma^{5}\right) / 2, \epsilon_{a}^{L}$ and $\epsilon_{a}^{R}$ are linear combinations of $\epsilon_{a}$ and $\tilde{\epsilon}_{a}$ :

$$
\begin{aligned}
\epsilon_{S} & =\frac{1}{2}\left(\epsilon_{S}^{L}+\epsilon_{S}^{R}\right), \quad \tilde{\epsilon}_{S}=\frac{i}{2}\left(\epsilon_{S}^{L}-\epsilon_{S}^{R}\right), \\
\epsilon_{P} & =\frac{i}{2}\left(\epsilon_{P}^{L}-\epsilon_{P}^{R}\right), \quad \tilde{\epsilon}_{P}=\frac{-1}{2}\left(\epsilon_{P}^{L}+\epsilon_{P}^{R}\right), \\
\epsilon_{V} & =\frac{1}{2}\left(\epsilon_{V}^{L}+\epsilon_{V}^{R}\right), \quad \tilde{\epsilon}_{V}=\frac{1}{2}\left(\epsilon_{V}^{R}-\epsilon_{V}^{L}\right), \\
\epsilon_{A} & =\frac{1}{2}\left(\epsilon_{A}^{R}-\epsilon_{A}^{L}\right), \quad \tilde{\epsilon}_{A}=\frac{1}{2}\left(\epsilon_{A}^{L}+\epsilon_{A}^{R}\right), \\
\epsilon_{T} & =\frac{1}{2}\left(\epsilon_{T}^{L}+\epsilon_{T}^{R}\right), \quad \tilde{\epsilon}_{S}=\frac{i}{2}\left(\epsilon_{T}^{L}-\epsilon_{T}^{R}\right) .
\end{aligned}
$$

Given the chiral structure of the SM, and the fact that most results are symmetric under $L \leftrightarrow R$, in this work we will adopt the chiral basis. Note that while $\epsilon_{a}$ and $\tilde{\epsilon}_{a}$ in eq. (2.1) are real and independent of each other, $\epsilon_{a}^{L}$ and $\epsilon_{a}^{R}$ in the chiral basis are either complex conjugate of each other $\left(\epsilon_{S}^{R}=\epsilon_{S}^{L *}, \epsilon_{P}^{R}=\epsilon_{P}^{L *}, \epsilon_{T}^{R}=\epsilon_{T}^{L *}\right)$, or real and independent $\left(\epsilon_{V}^{R}=\right.$ $\left.\operatorname{Re}\left[\epsilon_{V}^{R}\right], \epsilon_{V}^{L}=\operatorname{Re}\left[\epsilon_{V}^{R}\right], \epsilon_{A}^{R}=\operatorname{Re}\left[\epsilon_{A}^{R}\right], \epsilon_{A}^{L}=\operatorname{Re}\left[\epsilon_{A}^{R}\right]\right)$. Hence the full set of $\epsilon^{\prime}$ s in the chiral basis still contains 10 real independent parameters.

\subsection{DM relic abundance}

The relic abundance of $\chi$ via the standard freeze-out mechanism is approximately given by (see e.g. [41])

$$
\Omega_{\chi} h^{2} \simeq 0.12 \frac{x_{\text {f.o }}}{23} \frac{\sqrt{g_{\star}}}{10} \frac{1.7 \times 10^{-9} \mathrm{GeV}^{-2}}{\langle\sigma v\rangle},
$$

\footnotetext{
${ }^{3}$ This can be seen as follows. In the chiral basis, one can expand it as $\bar{\chi} \sigma^{\mu \nu} \gamma^{5} \chi \bar{f} \sigma_{\mu \nu} f=\left(-\overline{\chi_{R}} \sigma^{\mu \nu} \chi_{L}+\right.$ $\left.\overline{\chi_{L}} \sigma^{\mu \nu} \chi_{R}\right)\left(\overline{f_{R}} \sigma_{\mu \nu} f_{L}+\overline{f_{L}} \sigma_{\mu \nu} f_{R}\right)$. Since the cross terms vanish (according to Fierz transformations), $\overline{\chi_{R}} \sigma^{\mu \nu} \chi_{L} \bar{f}_{L} \sigma_{\mu \nu} f_{R}=\overline{\chi_{L}} \sigma^{\mu \nu} \chi_{R} \overline{f_{R}} \sigma_{\mu \nu} f_{L}=0$, the remaining terms imply $\bar{\chi} \sigma^{\mu \nu} \gamma^{5} \chi \bar{f} \sigma_{\mu \nu} f=\bar{\chi} \sigma^{\mu \nu} \chi \bar{f} \sigma_{\mu \nu} \gamma^{5} f$.
} 
where $x_{\text {f.o }} \equiv T_{\text {f.o }} / m_{\chi}$ is the ratio of the freeze-out temperature $T_{\text {f.o }}$ to the WIMP mass $m_{\chi} ; g_{\star}$ is the effective number of relativistic degrees of freedom in the thermal bath at freeze-out; and $\langle\sigma v\rangle$ is defined as [42, 43]

$$
\begin{aligned}
& \langle\sigma v\rangle \equiv n_{\mathrm{EQ}}^{-2} \int|\mathcal{M}|^{2} d \Pi_{1} d \Pi_{2} d \Pi_{3} d \Pi_{4}(2 \pi)^{4} \delta^{4} f_{1} f_{2}, \\
& n_{\mathrm{EQ}} \equiv \int 2 E_{1} d \Pi_{1} f_{1}, \quad d \Pi_{i} \equiv \frac{g_{i} d^{3} \mathbf{p}_{i}}{(2 \pi)^{3} 2 E_{i}} .
\end{aligned}
$$

Here subscripts $1,2, \cdots$, and 4 denote quantities of the first, second, $\cdots$, and the fourth particles in $\chi+\bar{\chi} \rightarrow f+\bar{f} ; \delta^{4}$ is short for $\delta^{4}\left(p_{1}+p_{2}-p_{3}-p_{4}\right) ; f_{1}\left(f_{2}\right)$ is the thermal distribution function of $\chi(\bar{\chi})$; and $g_{i}$ denotes the internal degree of freedom of the $i$-th particle. The squared amplitudes $|\mathcal{M}|^{2}$ has been evaluated and summarized in table 1 .

For $P, V, T$ interactions, the annihilation amplitudes are of the s-wave type and consequently are nearly constant in the non-relativistic regime. In this case, we can neglect the velocity dependence and reduce eq. (2.10) to

$$
\langle\sigma v\rangle \equiv \frac{1}{4 m_{\chi}^{2}} \int|\mathcal{M}|^{2} d \Pi_{3} d \Pi_{4}(2 \pi)^{4} \delta^{4}=\frac{|\mathcal{M}|^{2}}{32 \pi m_{\chi}^{2}} .
$$

For $S$ and $A$ interactions, we have $|\mathcal{M}|^{2} \propto v^{2}$ (p-wave annihilation) and hence the integration is somewhat more complicated. Assuming Maxwell-Boltzmann distributions for $f_{1}$ and $f_{2}$, eq. (2.10) can be reduced to [43]

$$
\langle\sigma v\rangle \equiv \frac{1}{8 m_{\chi}^{4} T K_{2}^{2}\left(m_{\chi} / T\right)} \int_{4 m_{\chi}^{2}}^{\infty} \sigma \sqrt{s}\left(s-4 m_{\chi}^{2}\right) K_{1}(\sqrt{s} / T) d s .
$$

Here $K_{1}$ and $K_{2}$ are $K$-type Bessel function of orders 1 and $2, s=\left(p_{1}+p_{2}\right)^{2}=4 m_{\chi}^{2}+m_{\chi}^{2} v^{2}$, and $\sigma$ is the total annihilation cross section [44]:

$$
\sigma=\int \frac{|\mathcal{M}|^{2}}{16 \pi s\left(s-4 m_{\chi}^{2}\right)} d q^{2},
$$

where $q^{2}=\left(p_{3}-p_{1}\right)^{2} \approx-m_{\chi}^{2}(1-v \cos \theta)$, with $\theta$ the angle between $\mathbf{p}_{1}$ and $\mathbf{p}_{3}$. Integrating $q^{2}$ from $-m_{\chi}^{2}(1+v)$ to $-m_{\chi}^{2}(1-v)$, we obtain results for $\sigma$ that are given in table 1 .

Plugging the results for $\sigma$ into eq. (2.13) with $v \rightarrow m_{\chi}^{-1} \sqrt{s-4 m_{\chi}^{2}}$, we can integrate eq. (2.13) analytically by noticing that for any value of $p>-1$,

$$
\int_{4 m^{2}}^{\infty}\left(s-4 m^{2}\right)^{p} K_{1}(\sqrt{s} / T) \frac{1}{\sqrt{s}} d s=2^{1+2 p} T(m T)^{p} K_{p}(2 m / T) \Gamma(1+p),
$$

where $\Gamma$ is the Euler gamma function. The results for $\langle\sigma v\rangle$ are then expanded in $T / m$ and summarized in table 1.

Using the results for $\langle\sigma v\rangle$ with $T_{\text {f.o }} \simeq m_{\chi} / 23$ (the typical freeze-out temperature) in eq. (2.9), we obtain

$$
\Omega_{\chi} h^{2} \simeq 0.12\left(\frac{100 \mathrm{GeV}}{m_{\chi}}\right)^{2}\left|\frac{\epsilon_{a}^{\star}}{\epsilon_{a}^{L, R}}\right|^{2},
$$




\begin{tabular}{|cccccc|}
\hline & $S$ & $P$ & $V$ & $A$ & $T$ \\
\hline$|\mathcal{M}|^{2} /\left|G_{F} m_{\chi}^{2} \epsilon_{a}^{L, R}\right|^{2}$ & $2 v^{2}$ & 8 & 16 & $2 v^{2}\left(1+\cos ^{2} \theta\right)$ & 32 \\
$\sigma /\left|G_{F} m_{\chi}^{2} \epsilon_{a}^{L, R}\right|^{2}$ & $\frac{v}{4 \pi s}$ & $\frac{1}{\pi s v}$ & $\frac{2}{\pi s v}$ & $\frac{v}{3 \pi s}$ & $\frac{4}{\pi s v}$ \\
$\langle\sigma v\rangle /\left|G_{F} m_{\chi}^{2} \epsilon_{a}^{L, R}\right|^{2}$ & $\frac{3}{8 \pi} T m_{\chi}^{-3}$ & $\frac{1}{4 \pi} m_{\chi}^{-2}$ & $\frac{1}{2 \pi} m_{\chi}^{-2}$ & $\frac{1}{2 \pi} T m_{\chi}^{-3}$ & $\frac{1}{\pi} m_{\chi}^{-2}$ \\
$\epsilon_{a}^{\star}$ & 0.49 & 0.13 & 0.089 & 0.43 & 0.063 \\
\hline
\end{tabular}

Table 1. Annihilation amplitudes $\left(|\mathcal{M}|^{2}\right)$, cross sections $(\sigma)$, thermally averaged cross sections $(\langle\sigma v\rangle)$, and benchmark values of $\epsilon_{a}^{\star}$ used in eq. (2.16) for the five types of effective interactions. We neglected the mass of the final states and assume that the annihilating DM particles are nonrelativistic with $v$ being their relative velocity. The results have been expanded in $v$ and only leading-order terms are retained. Results for $\epsilon_{a}^{R}$ and $\epsilon_{a}^{L}$ are identical.

where $\epsilon_{a}^{\star}$ denotes benchmark values: $\epsilon_{S}^{\star}=0.49, \epsilon_{P}^{\star}=0.13, \epsilon_{V}^{\star}=0.089, \epsilon_{A}^{\star}=0.43$, and $\epsilon_{T}^{\star}=0.063$. Note that $\epsilon_{a}^{\star}$ for $a=S$ or $A$ is generally larger than for other cases because the cross section is velocity suppressed, which implies that they would freeze out at higher temperatures for the same coupling strength. Hence to reach the same relic abundance (i.e. same freeze out temperature), the coupling needs to be larger.

\section{Loop-induced electromagnetic interactions}

In the presence of any of the $\bar{\chi}-\chi-\bar{f}-f$ interactions formulated in eq. (2.1) or eq. (2.3), we can close the fermion lines of $f$ and attach a photon external line, as illustrated in figure 1. This generally leads to loop-induced electromagnetic interactions of $\chi$.

By closing the loop, one gets an amplitude which takes the general form,

$$
i \mathcal{M}_{\text {loop }}=i \overline{u_{2}} \mathcal{F}^{\mu} u_{1} \varepsilon_{\mu},
$$

where $\overline{u_{2}}, u_{1}, \varepsilon_{\mu}$ represent the three external lines and $\mathcal{F}^{\mu}$ denotes the vertex function. The most general form of $\mathcal{F}^{\mu}$ that respects Lorentz and electromagnetic gauge invariance can be decomposed as a combination of four terms, each one with its own form factor (see e.g. $[45,46])$ :

$$
\mathcal{F}^{\mu}=\mathcal{F}_{Q}\left(q^{2}\right) \gamma^{\mu}+\mathcal{F}_{M}\left(q^{2}\right) i \sigma^{\mu \nu} q_{\nu}+\mathcal{F}_{E}\left(q^{2}\right) \sigma^{\mu \nu} \gamma^{5} q_{\nu}+\mathcal{F}_{A}\left(q^{2}\right)\left(q^{2} \gamma_{\mu}-q_{\mu} q\right) \gamma^{5},
$$

where $q \equiv p_{1}-p_{2}$ is the photon momentum. In the limit of $q^{2} \rightarrow 0$, the four form factors $\mathcal{F}_{Q}(0), \mathcal{F}_{M}(0), \mathcal{F}_{E}(0)$, and $\mathcal{F}_{A}(0)$ correspond to the electric charge, magnetic dipole, electric dipole and anapole of $\chi$, respectively. For simplicity, we denote

$$
d_{M} \equiv \mathcal{F}_{M}(0), \quad d_{E} \equiv \mathcal{F}_{E}(0)
$$

In this work, we do not consider the electric charge and anapole of $\chi$ because the former remains zero at loop levels if DM is electrically neutral at tree level and the latter causes suppressed signals in DM direct detection. This suppression can be seen from the form 



Figure 1. Loop-induced electromagnetic interactions. In the presence of effective $\bar{\chi}-\chi-\bar{f}$ - $f$ interactions where $\chi$ is a DM fermion and $f$ is a SM fermion, the $f$ and $\bar{f}$ lines can be closed to form a loop diagram, which after attaching a photon line can generate electric and/or magnetic dipoles of DM. The UV structure of the effective vertex, which is important for the loop-induced dipoles, in simplified models typically can be one of the four diagrams, (a)-(d).

of the $\mathcal{F}_{A}$ term of eq. (3.2), which in the low- $q^{2}$ regime is proportional to $q^{2}$. This $\mathcal{O}\left(q^{2}\right)$ coefficient will be canceled by the photon propagator which is proportional to $\mathcal{O}\left(q^{-2}\right)$. Indeed, ref. [28] has shown that the effect of anapole in direct detection is nearly equivalent to that of contact interactions. Thus, unlike with dipoles, the direct detection does not profit from the several orders of magnitude enhancement related to the $1 / q^{2}$ behavior of the amplitude, see below. Neutral $\chi$ might possess a non-vanishing charge radius defined as $d \mathcal{F}_{Q}\left(q^{2}\right) /\left.d q^{2}\right|_{q^{2} \rightarrow 0}$. Its effect in direct detection is also suppressed for the same reason. ${ }^{4}$

As emphasized in the introduction, in this section we will compute the dipoles resulting from figure 1 at various levels. First we will simply consider the loop diagrams over the 4-fermion interactions. In the second step (section 3.2) we replace the constant dimensional coupling of the 4-fermion interactions by an energy-dependent one that behaves like an $s$ or $t$-channel propagator. By performing the calculation for each operator, we obtain the dipole "building blocks" for the cases where the contact interactions would be induced at tree level. These are building blocks in the sense that considering explicit scalar or vector boson mediator setups, which will be considered in the third step (see section 3.3), the dipole results are simply some combinations of these building block results obtained in section 3.2. Finally we consider examples of UV complete model calculations in section 3.4 and compare with previous results.

\footnotetext{
${ }^{4}$ For a scalar DM candidate the coupling to the photon that could be loop induced from an effective operator (i.e. $\phi_{D M}^{\dagger} \phi_{D M} \bar{f} f$ or $\phi_{D M}^{\dagger} \phi_{D M} \bar{f} \gamma_{5} f$ ), coupling it in pairs to a pair of charged fermions, would lead to suppressed direct detection in a similar way, as it would induce only a charged radius. For a Majorana DM candidate, as is well known, dipole interactions identically vanish, and an anapole leads to suppressed direct detection in a similar way than for a Dirac fermion.
} 


\subsection{Loop integrals with contact interactions}

Assuming that the blue blob in figure 1 is a contact interaction which is independent of the loop momentum, the amplitude of the loop diagram reads

$$
i \mathcal{M}_{\text {loop }}=i \overline{u_{2}} \Gamma^{a} u_{1} \int \frac{d^{4} k}{(2 \pi)^{4}} \operatorname{tr}\left[\frac{1}{\not k_{2}-m_{f}} i e Q_{f} \gamma^{\mu} \frac{1}{\not k_{1}-m_{f}} \tilde{\Gamma}^{a}\right] G_{X} \varepsilon_{\mu}
$$

where $\Gamma^{a}$ and $\tilde{\Gamma}^{a}$ denote possible Dirac matrices according to eq. (2.3), $k=p_{1}-k_{1}=p_{2}-k_{2}$ with $p_{1,2}$ and $k_{1,2}$ already indicated in figure 1 , and $G_{X}$ denotes the strength of the fourfermion effective interaction. A straightforward loop calculation with a constant $G_{X}$ gives

$$
i \mathcal{M}_{\text {loop }} \stackrel{\text { constant } G_{X}}{\longrightarrow} \begin{cases}d_{M}, d_{E}=0 & \text { for } a=S, P, V, A \\ d_{M}, d_{E}=\text { divergent } & \text { for } a=T\end{cases}
$$

The result can be understood as follows. Factorizing out the photon polarization 4-vector $\epsilon_{\mu}$, by construction the integral must be proportional to the external 4-momentum, but for all $S, P, V$ and $A$ cases the loop integral presents a shift symmetry which means that the result is the same as in the case that the photon has vanishing 4-momentum. Only for the tensor case do we get a non vanishing result. However in this case the result we get is divergent:

$$
\left(d_{M}, d_{E}\right)=\frac{e Q_{f} G_{F}}{4 \pi^{2}}\left(\epsilon_{T}^{L}+\epsilon_{T}^{R},-i \epsilon_{T}^{L}+i \epsilon_{T}^{R}\right) m_{f} C^{(s)} \quad \text { for } a=T,
$$

where $C^{(s)} \equiv \frac{1}{\varepsilon}+\log \left(\mu^{2} / m_{f}^{2}\right)$, and $\mu$ and $\varepsilon$ are defined by dimensional regularization: $d^{4} k /(2 \pi)^{4} \rightarrow \mu^{2 \varepsilon} d^{4-2 \varepsilon} k /(2 \pi)^{4-2 \varepsilon}$. This divergence can be cancelled by divergences coming from additional loop contributions that should be present when integrating out the heavy states in a given UV complete theory (for instance from loop contributions of other operators involving not only the 4 fermions but also the photon field, see the discussion towards the end of the appendix). This shows that to consider an EFT with only the 4-fermion interactions as we do in this subsection is not consistent. Other operators contributing to the dipoles at one loop must necessarily also exist in some cases in a fully consistent EFT treatment, bringing additional finite contributions. However from the low energy point of view the coefficients of these operators are unknown, unlike the one of the 4 -fermion interaction which is assumed to dominate the DM freeze-out process.

\subsection{Including $t$ - and $s$-channel energy dependence}

The fact that considering only the loop controbutions over the 4-fermion interactions we got vanishing dipoles for the $S, P, V$ and $A$-fermion operators could suggest that it is hopeless to get observable effects related to these operators via the dipoles they induce. However we would like to stress that this is not true at all. Different ways to induce the same 4-fermion operators after integrating out the heavy states, can give different results for figure 1 (i.e. when not integrating out the heavy fields). To show that, let us consider possible UV behavior of the effective interactions, which usually originate from integrating 
an $s$ - or $t$-channel propagator. Hence, instead of considering $G_{X}$ as a constant in eq. (3.4), we consider plugging the following two forms of $G_{X}$ with $s$ - or $t$-channel energy dependence:

$$
G_{X}^{(t)}=\frac{1}{t-m_{\phi}^{2}}, G_{X}^{(s)}=\frac{1}{s-m_{\phi}^{2}}
$$

where $t=\left(p_{1}-k_{1}\right)^{2}$ and $s=\left(p_{1}-p_{2}\right)^{2}$ are Mandelstam variables ${ }^{5}$ and $m_{\phi}$ is an energy scale which will be related to a mediator mass. Performing the loop integration with the above energy dependence included, we obtain the results in table 2. As is shown in this table, although the two cases at low energies lead to the same four-fermion operators, they do not lead to the same dipoles. For the $s$-channel case one gets the same results as for the contact interaction cases in section 3.1. In particular one gets vanishing results for the $S, P, V$ and $A$ cases because introducing the $s$-channel energy dependence does not spoil the momentum shift symmetry in the loop integral. This can also be understood by noting that the diagram in this case contains a self-energy loop which cannot give rise to a $\sigma_{\mu \nu}$. For the tensor case instead one can get a dipole as the effective operator already contain a $\sigma_{\mu \nu}$ to start with. It is divergent as in the contact interaction case. ${ }^{6}$

Instead, for the $t$-channel case a magnetic or an electric dipole is always induced (even if never both), depending on the operator considered. In all cases this allows non-suppressed direct detection signals as we will see below. Baring cancellations this implies that any UV complete model generating any one of these operators through a $t$-channel transition can be efficiently probed via direct detection, see below. This is presumably also the case for models where the effective interactions would be induced at loop level, such as through box diagrams (but we will not explicitly check this statement here). Note that for this $t$-channel case all the results are obtained finite. It is noteworthy that the loop-induced dipoles for the $S, P$ and $T$ cases are proportional to $m_{f}$ while for the other two cases they are proportional to $m_{\chi}$. This is due to the well-known chirality-flipping nature of $S, P, T$ interactions - see discussions in ref. [47].

In summary, inserting the energy-dependent ( $s$ - or $t$-channel) $G_{X}$, we get either vanishing or non-vanishing dipoles. The non-vanishing dipoles are given by

$$
d_{M, E}=\frac{e Q_{f} G_{F}}{16 \pi^{2}} \times \mathcal{O}\left(\epsilon_{a}\right) \times\left\{\begin{array}{ll}
m_{\chi} & \text { for } a=V, A \\
m_{f} & \text { for } a=S, P, T
\end{array},\right.
$$

where the $\mathcal{O}\left(\epsilon_{a}\right)$ part has been specified in table 2 .

\footnotetext{
${ }^{5}$ Note that when the tree-level diagrams are interpreted as DM annihilation,we flip the direction of $p_{2}$ and obtain $s=\left(p_{1}+p_{2}\right)^{2}$ which is the conventional definition of $s$ as the Mandelstam variable.

${ }^{6}$ Note that the tensor operator cannot result from a simple tree level $s$-channel exchange, but must be induced e.g. from a loop diagram coupling the $s$-channel mediator to the pair of DM particles and from another loop diagram coupling the $s$-channel mediator to the pair of SM fermions. This case is thus of limited interest. Tensor interactions are generated in an easier way from tree level $t$-channel (through Fierz transformation) or one loop box diagrams. Thus we will not elaborate more on this case. At a very rough level one can expect constraints on this case similar to the ones obtained below for the $t$-channel $T$ case.
} 


\begin{tabular}{|cccccc|}
\hline & $S$ & $P$ & $V$ & $A$ & $T$ \\
\hline$d_{M}^{(t)} /\left(\frac{e Q_{f} G_{F}}{16 \pi^{2}}\right)$ & $\left(\epsilon_{S}^{L}+\epsilon_{S}^{R}\right) m_{f}$ & 0 & $\frac{1}{3}\left(\epsilon_{V}^{L}+\epsilon_{V}^{R}\right) m_{\chi}$ & $-\left(\epsilon_{A}^{L}-\epsilon_{A}^{R}\right) m_{\chi}$ & $4\left(\epsilon_{T}^{L}+\epsilon_{T}^{R}\right) m_{f} C^{(t)}$ \\
$d_{E}^{(t)} /\left(\frac{e Q_{f} G_{F}}{16 \pi^{2}}\right)$ & 0 & $-\left(\epsilon_{P}^{L}+\epsilon_{P}^{R}\right) m_{f}$ & 0 & 0 & $-4 i\left(\epsilon_{T}^{L}-\epsilon_{T}^{R}\right) m_{f} C^{(t)}$ \\
$d_{M}^{(s)} /\left(\frac{e Q_{f} G_{F}}{16 \pi^{2}}\right)$ & 0 & 0 & 0 & 0 & $4\left(\epsilon_{T}^{L}+\epsilon_{T}^{R}\right) m_{f} C^{(s)}$ \\
$d_{E}^{(s)} /\left(\frac{e Q_{f} G_{F}}{16 \pi^{2}}\right)$ & 0 & 0 & 0 & 0 & $-4 i\left(\epsilon_{T}^{L}-\epsilon_{T}^{R}\right) m_{f} C^{(s)}$ \\
\hline
\end{tabular}

Table 2. Loop-induced electromagnetic dipoles obtained for the various operators assuming a $t$ or s-channel energy dependence for their coupling strengths. Here $d_{M}^{(t)}$ and $d_{E}^{(t)}$ are generated by the $t$-channel $G_{X}^{(t)}$, and $d_{M}^{(s)}$ and $d_{E}^{(s)}$ by the $s$-channel $G_{X}^{(s)}$ - see in eq. (3.7). $C^{(t)}$ and $C^{(s)}$ are given by $C^{(t)} \equiv 1+\log \left(m_{f}^{2} / m_{\phi}^{2}\right), \quad C^{(s)} \equiv \frac{1}{\varepsilon}+\log \left(\mu^{2} / m_{f}^{2}\right)$, with $\mu$ and $\varepsilon$ defined by the dimensional regularization $d^{4} k /(2 \pi)^{4} \rightarrow \mu^{2 \varepsilon} d^{4-2 \varepsilon} k /(2 \pi)^{4-2 \varepsilon}$.

\subsection{Simplified models with explicit mediators}

In the previous subsection we computed the dipoles obtained for each four-fermion operator with $s$ - or $t$-channel energy dependence. In this subsection we consider simplified models (in analogy to the concept of simplified models in collider searches for DM [48-54]) with explicit mediators that do induce these operators via $t$ or $s$-channel exchange of scalar or vector boson mediators. The results we get for these simplified models are combination of the ones obtained in the previous subsection for the $S, P, V, A$ and $T$ operators. These results remain "simplified" because they are limited to the loop contribution of diagrams where the photon is attached to the charged fermion propagator.

At tree level, there are four simple ways to open the four-fermion operator interactions, via (a) a $t$-channel scalar mediator, (b) a $t$-channel vector mediator, (c) an s-channel scalar mediator, (d) an $s$-channel vector mediator - all are presented in figure 1. There could be more complex ways. For instance, the effective vertex could be generated by a box diagram, which is beyond the scope of this work.

Let us denote the scalar mediator by $\phi$ and the vector mediator by $\phi^{\mu}$. The most general couplings for cases (a)-(d) can be formulated as follows

$$
\mathcal{L} \supset \begin{cases}\bar{\chi} \phi\left(g_{L} P_{L}+g_{R} P_{R}\right) f+\text { h.c. } & \text { case (a) } \\ \bar{\chi} \gamma^{\mu} \phi_{\mu}\left(g_{L} P_{L}+g_{R} P_{R}\right) f+\text { h.c. } & \text { case (b) } \\ \bar{\chi}\left(g_{L, \chi} P_{L}+g_{R, \chi} P_{R}\right) \chi \phi+(\chi \rightarrow f) & \text { case (c) } \\ \bar{\chi} \gamma^{\mu} \phi_{\mu}\left(g_{L, \chi} P_{L}+g_{R, \chi} P_{R}\right) \chi+(\chi \rightarrow f) & \text { case (d) }\end{cases}
$$

where $g_{L}$ and $g_{R}$ are two generic couplings. They can be complex numbers. For example, pseudo-scalar couplings corresponds to $\operatorname{Re}\left(g_{L}\right)=\operatorname{Re}\left(g_{R}\right)=0$ and $\operatorname{Im}\left(g_{L}\right)=-\operatorname{Im}\left(g_{R}\right) \neq 0$.

Attaching an external photon to the charged fermion in the loop, and performing the loop integral, the results we obtain for cases (a)-(d), are given in table 3.

For cases (a) and (b) which are $t$-channel, we find that the Lagrangian generally generates magnetic and electric dipoles $\left(d_{M}\right.$ and $\left.d_{E}\right)$, depending on the following combinations of couplings:

$$
g^{2} \equiv g_{L} g_{L}^{*}+g_{R} g_{R}^{*}, \quad g_{+}^{2} \equiv g_{L} g_{R}^{*}+g_{R} g_{L}^{*}, \quad g_{-}^{2} \equiv i\left(g_{L} g_{R}^{*}-g_{R} g_{L}^{*}\right) .
$$




\begin{tabular}{|ccc|}
\hline case & $d_{M} /\left(\frac{e Q_{f}}{16 \pi^{2} m_{\phi}^{2}}\right)$ & $d_{E} /\left(\frac{e Q_{f}}{16 \pi^{2} m_{\phi}^{2}}\right)$ \\
\hline (a) & $-g_{+}^{2} m_{f}\left(\frac{3}{4}+\ln \frac{m_{f}}{m_{\phi}}\right)+\frac{1}{6} m_{\chi} g^{2}$ & $g_{-}^{2} m_{f}\left(\frac{3}{4}+\ln \frac{m_{f}}{m_{\phi}}\right)$ \\
(b) & $-g_{+}^{2} m_{f}+\frac{2}{3} m_{\chi} g^{2}$ & $-g_{-}^{2} m_{f}$ \\
(c) or (d) & 0 & 0 \\
\hline
\end{tabular}

Table 3. Electromagnetic dipoles generated by the loop diagram in figure 1 for cases (a)-(d).

For cases (c) and (d) which are $s$-channel, we find that the above Lagrangian always leads to vanishing dipoles. The reason for this has been previously discussed in section 3.2. For case (c), one can note in addition that the loop would imply an effective mixing $\phi \partial^{\mu} A_{\mu}$ (or equivalently $\partial^{\mu} \phi A_{\mu}$ ) between the scalar $\phi$ and the photon $A_{\mu}$, which has to be zero given that the photon has no longitudinal polarization. For case (d), the Lorentz structure of the loop can be generally written as $g_{\mu \nu} \Pi_{1}+q_{\mu} q_{\nu} \Pi_{2}$ where $\Pi_{1}$ and $\Pi_{2}$ are $q^{2}$-dependent scalar quantities. Following the notations in eq. (3.1), when combined with $\overline{u_{2}} \gamma^{\mu} u_{1}$ (assuming $\left.g_{L, \chi}=g_{R, \chi}\right)$, only the $g_{\mu \nu} \Pi_{1}$ part remains due to $\overline{u_{2}} q u_{1}=0$. Hence it only contributes to the form factor $\mathcal{F}_{Q}$ in eq. (3.2) and only gives rise to the charge radius of $\chi$, not dipoles. If $\overline{u_{2}} \gamma^{\mu} u_{1}$ is replaced with $\overline{u_{2}} \gamma^{\mu} \gamma^{5} u_{1}$, then it gives rise to $\mathcal{F}_{A}$, the anapole of $\chi$.

The results obtained for simplified models, table 3 , are combinations of the results obtained using energy-dependent effective interactions, table 2 of section 3.2, via some Fierz transformations. To show that let us integrate out $\phi\left(\right.$ or $\left.\phi^{\mu}\right)$ in eq. (3.9) we obtain the following four-fermion interactions:

$$
\mathcal{L}_{\text {eff }}=\frac{1}{m_{\phi}^{2}} \bar{\chi} \Gamma\left(g_{L} f_{L}+g_{R} f_{R}\right)\left(g_{L}^{*} \overline{f_{L}}+g_{R}^{*} \overline{f_{R}}\right) \Gamma \chi,
$$

where $\Gamma=1$ or $\gamma^{\mu}$ for case (a) or (b) respectively. Performing Fierz transformations, ${ }^{7}$ the effective interaction for case (a) can be expressed in the form of eq. (2.3) with the $\epsilon$ 's given by

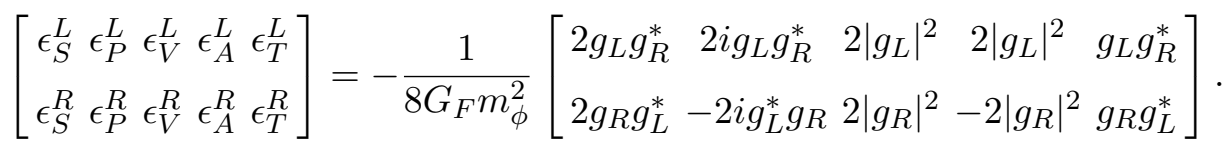

For case (b), the corresponding $\epsilon$ 's are given by

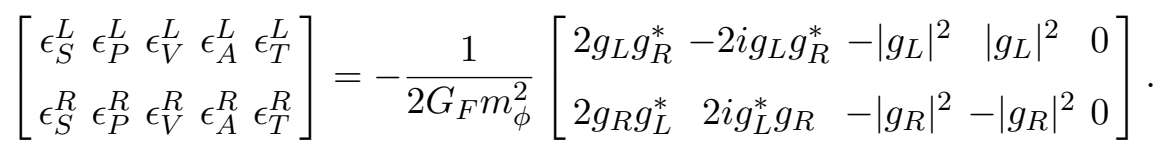

Using eqs. (3.12)-(3.13) and table 2, one can immediately see (with the replacement $\left.g^{2} / m_{\phi}^{2} \rightarrow \epsilon G_{F}\right)$ that the dipoles obtained in this way are exactly identical to those in table 3. For example, according to table 2, only $V$ and $A$ interactions leads to $d_{M} \propto m_{\chi}$. Hence the $m_{\chi}$ term in $d_{M}$ for case (a) should be proportional to

$$
\left(\frac{1}{3} \epsilon_{V}^{L}+\frac{1}{3} \epsilon_{V}^{R}-\epsilon_{A}^{L}+\epsilon_{A}^{R}\right) m_{\chi} \propto \frac{1}{6} m_{\chi}\left(\left|g_{L}\right|^{2}+\left|g_{R}\right|^{2}\right),
$$

which is consistent with table 3 .

\footnotetext{
${ }^{7}$ We use the coefficients in ref. [55], page 65 .
} 
Table 2 can also be used to show that the electric dipoles in table 3 should be proportional to $m_{f}$ only. This is because according to table 2 electric dipoles can only be generated by $P$ and $T$ interactions, which as previously mentioned always leads to $d_{E} \propto m_{f}$.

\subsection{UV complete example}

The results obtained via the simplified model approach in section 3.3 may still differ from what one would get in a UV complete model. Since DM is neutral and the SM fermions are charged, the $t$-channel heavy mediator has necessarily a non-vanishing electric charge. Thus in a UV complete model there are necessarily extra diagrams in which the photon is attached to the heavy mediator instead of the SM charged fermion. This implies that in the low energy limit the UV complete model also induces dipole contributions which do not reduce to a loop over the 4 -fermion interaction. However, unlike for the electric charge operator induced at one loop (for which charge conservation enforces the cancellation of these diagrams, see the end of the appendix) we do not expect in general that for dipoles these extra diagrams could induce any large destructive interference for the dipole induced (given in particular the chiral structure of the SM), or even largely change the results. To illustrate this, we consider one example of the many possibilities listed in eq. (3.9), i.e. the (a) model with couplings to right-handed SM charged fermion:

$$
\mathcal{L} \supset g_{R} \bar{\chi} f_{R} \phi+\text { h.c. }
$$

By integrating out the heavy scalar boson, this interaction induces the following 4-fermion interaction

$$
\mathcal{L}_{\text {eff }}=-G_{X} \bar{\chi} P_{R} f \bar{f} P_{L} \chi,
$$

where $G_{X}=g_{R} g_{R}^{*} / m_{\phi}^{2}$. One can reformulate it to the form in eq. (2.1) via Fierz transformation: ${ }^{8}$

$$
\mathcal{L}_{\text {eff }}=\frac{1}{4} G_{X} \bar{\chi} \gamma^{\mu} \chi \overline{f_{R}} \gamma_{\mu} f_{R}-\frac{1}{4} G_{X} \bar{\chi} \gamma^{\mu} \gamma^{5} \chi \overline{f_{R}} \gamma_{\mu} f_{R}
$$

Eq. (3.17) contains two types ( $V$ and $A$ ) of effective interactions, with the following $\epsilon$ 's:

$$
\begin{aligned}
\epsilon_{V}^{L} & =0, \epsilon_{V}^{R}=\frac{1}{4} G_{X} / G_{F}, \\
\epsilon_{A}^{L} & =0, \epsilon_{A}^{R}=-\frac{1}{4} G_{X} / G_{F} .
\end{aligned}
$$

According to section 3.3, the loop diagram obtained by closing the fermion line and attaching an external photon line leads to

$$
d_{M}^{(f)}=-\frac{1}{6} m_{\chi} \cdot \frac{e\left|g_{R}\right|^{2} Q_{f}}{16 \pi^{2} m_{\phi}^{2}},
$$

where the superscript $(f)$ indicates that it is from the $f$-photon coupling. Alternatively, one can use Fierz transformations and table 2 in a way similar to the calculation from eq. (3.11) to eq. (3.13) to obtain the dipole. This leads to exactly the same result.

\footnotetext{
${ }^{8}$ See, e.g., ref. [55], page 65.
} 
The other diagram with the photon coupled to $\phi$ leads to (see appendix A for the calculation):

$$
d_{M}^{(\phi)}=\frac{1}{12} m_{\chi} \cdot \frac{e\left|g_{R}\right|^{2} Q_{\phi}}{16 \pi^{2} m_{\phi}^{2}},
$$

where $Q_{\phi}$ is the electric charge of $\phi$.

Note that each of these diagrams gives a finite contribution to the dipoles but a UV divergent contribution to the electric charge operator. According to eqs. (A.12) and (A.13), the divergent part of the total amplitude reads:

$$
\mathcal{M}^{\text {divergent }}=\frac{e\left|g_{R}\right|^{2}}{16 \pi^{2}} \overline{u_{2}} \gamma^{\mu} u_{1} \varepsilon_{\mu}\left(\frac{1}{2 \epsilon} Q_{f}+\frac{1}{2 \epsilon} Q_{\phi}\right),
$$

which cancels out due to electric charge conservation, $Q_{\phi}+Q_{f}=0$.

Combining eqs. (3.19) and (3.20) with $Q_{\phi}=-Q_{f}$, the total contribution to the magnetic dipole reads:

$$
d_{M}=-\frac{1}{4} m_{\chi} \cdot \frac{e\left|g_{R}\right|^{2} Q_{f}}{16 \pi^{2} m_{\phi}^{2}} .
$$

We see that the actual magnetic dipole is $50 \%$ higher than the result obtained using table 2 or table 3. Therefore, when using table 2 or table 3, one should keep in mind that the results may be changed due to new contributions in UV complete calculations (from the EFT point of view, they correspond to loop contributions on extra operators whose coefficients are a priori unknown). Nevertheless, the results of table 2 and table 3, which are based on considerations starting from effective four-fermion operators (whose coefficients can be estimated from the relic density constraint), provide correct estimates of the order of magnitude of the dipoles. In other words, one cannot exclude that specific UV models would give quite different results between both approaches but this explicit example shows that in simple frameworks this is not the case.

Note that, as another example of UV model, a purely left-handed interaction involving a SM fermion doublet and a scalar doublet instead of $f_{R}$ and $\phi^{+}$in eq. (3.15) gives the same dipoles as the purely right-handed case of eq. (3.15). Note also that for s-channel UV complete models the mediator is neutral and the photon cannot be attached to the mediator. Hence there is no such extra diagram. Thus for the (c) and (d) models above the full UV complete results are the same as the ones obtained in section 3.3.

\section{Expected magnitude of electromagnetic dipoles}

The relic abundance constraint, $\Omega_{\chi} h^{2} \simeq 0.12$, requires that the $\epsilon$ coefficients of the effective operators are typically of the order of a few (or tens of ) percent - see table 1 and eq. (2.16). By requiring that $\Omega_{\chi} h^{2} \simeq 0.12$ is correctly produced, according to eq. (2.16), we can obtain expected values for the dipoles. We will do that at the level of the building blocks of section 3.2. By replacing $\epsilon_{a}^{L, R}$ in table 2 with $100 \mathrm{GeV} \cdot m_{\chi}^{-1} \epsilon_{a}^{\star}$, we obtain

$$
\left|d_{M}^{(t)}\right| \approx\left\{\begin{array}{ll}
4.3 \times 10^{-21}\left|Q_{f}\right| \text { ecm } & \text { for } a=V \\
6.2 \times 10^{-20}\left|Q_{f}\right| \text { ecm } & \text { for } a=A
\end{array},\right.
$$


where ecm $\equiv e \times \mathrm{cm} \approx 15350.3 \mathrm{eV}^{-1}$ is a commonly used unit for electromagnetic dipoles. The result is almost independent of $m_{\chi}$ and $m_{f}$. It only depends on the electric charge of the SM fermion involved, $Q_{f}$, which can be $2 / 3$ (for $f=u, c, t$ ), $-1 / 3$ (for $f=d, s, b$ ), or -1 (for $f=e, \mu, \tau)$.

For $S, P$, and $T$ interactions, the dipoles depend on $m_{f}$. Since in this work we require that $\chi \bar{\chi} \rightarrow f \bar{f}$ is responsible for the relic abundance, we concentrate on cases with $m_{\chi} \gtrsim m_{f}$. With this assumption, one can still apply $\epsilon_{a}^{L / R}=100 \mathrm{GeV} \cdot m_{\chi}^{-1} \epsilon_{a}^{\star}$ to the remaining dipoles:

$$
\begin{aligned}
\left|d_{M}^{(t)}\right| & \approx 7.2 \times 10^{-20}\left|Q_{f}\right| \frac{m_{f}}{m_{\chi}} \mathrm{ecm}, \quad \text { for } a=S, \\
\left|d_{E}^{(t)}\right| & \approx 1.8 \times 10^{-20}\left|Q_{f}\right| \frac{m_{f}}{m_{\chi}} \mathrm{ecm}, \quad \text { for } a=P, \\
\left|d_{E, M}^{(t)}\right| & \approx 3.7 \times 10^{-20}\left|Q_{f}\right| \frac{m_{f}}{m_{\chi}} \mathrm{ecm}, \quad \text { for } a=T .
\end{aligned}
$$

\section{Electromagnetic dipoles in direct detection}

In direct detection experiments, the differential event rate of DM-nucleus scattering ${ }^{9}$ can be evaluated via (see e.g. $[44,56])$ :

$$
\frac{d R}{d E_{r}}=N_{T} n_{\chi} \epsilon\left(E_{r}\right) \int \frac{d \sigma}{d E_{r}} v f_{\oplus}(\mathbf{v}) \Theta\left(v-v_{\min }\right) d^{3} \mathbf{v} .
$$

Here $E_{r}$ is the nuclear recoil energy; $N_{T}$ is the total number of target nuclei; $n_{\chi}$ is the local DM number density; $\epsilon\left(E_{r}\right)$ is the detection efficiency; $\frac{d \sigma}{d E_{r}}$ is the differential cross section; $f_{\oplus}(\mathbf{v})$ is the DM velocity distribution in the Earth frame; $v_{\min }$ is the minimal velocity to generate a given $E_{r}$,

$$
v_{\min }=\sqrt{\frac{m_{N} E_{r}}{2 \mu_{\chi N}^{2}}}
$$

where $m_{N}$ is the nucleus mass and $\mu_{\chi N} \equiv m_{\chi} m_{N} /\left(m_{\chi}+m_{N}\right)$ is the DM-nucleus reduced mass. For the local DM density we take $n_{\chi}=\rho_{\chi} / m_{\chi}$ and $\rho_{\chi}=0.4 \mathrm{GeV} / \mathrm{cm}^{3}$ [57].

The DM velocity distribution $f_{\oplus}(\mathbf{v})$ is often parametrized by a truncated Maxwellian distribution in the frame of the Galaxy and then boosted to the Earth frame. The specific form reads

$$
f_{\oplus}(\mathbf{v})=\frac{1}{N_{f}} \exp \left[-\frac{\tilde{v}^{2}}{v_{0}^{2}}\right] \Theta\left(v_{\mathrm{esc}}-\tilde{v}\right),
$$

where $\tilde{v}=\left|\mathbf{v}+\mathbf{v}_{\oplus}\right|$ and $\left|\mathbf{v}_{\oplus}\right| \approx 240 \mathrm{~km} / \mathrm{s}$ is the velocity of the Earth with respect to the Galaxy; $v_{\mathrm{esc}} \approx 550 \mathrm{~km} / \mathrm{s}$ is the escape velocity of the Galaxy; $v_{0}=220 \mathrm{~km} / \mathrm{s}$ is the mean velocity of the Maxwellian distribution. The $N_{f}$ factor normalizes $f_{\oplus}$ so that $\int f_{\oplus}(\mathbf{v}) d^{3} \mathbf{v}=1:$

$$
N_{f}=\pi^{3 / 2} v_{0}^{3}\left[\operatorname{erf}\left(\frac{v_{\mathrm{esc}}}{v_{0}}\right)-\frac{2}{\sqrt{\pi}} \frac{v_{\mathrm{esc}}}{v_{0}} \exp \left(-\frac{v_{\mathrm{esc}}^{2}}{v_{0}^{2}}\right)\right] .
$$

\footnotetext{
${ }^{9}$ In ref. [16], it was pointed out for leptophilic DM that DM-nucleus scattering largely dominates over DM-electron scattering (for DM masses beyond $\mathrm{GeV}$ ) and this is expected here too for the same reasons.
} 
The differential cross sections for DM-nucleus scattering via dipoles read [17, 21]:

$$
\begin{aligned}
\frac{d \sigma_{M}}{d E_{r}} & \approx d_{M}^{2} \frac{\alpha Z^{2} F_{E}^{2}}{E_{r}}\left[1-\frac{E_{r}}{2 m_{N} v^{2}}\left(1+2 \frac{m_{N}}{m_{\chi}}\right)\right]+d_{M}^{2} \frac{\alpha G F_{M}^{2}}{2 m_{N} v^{2}}, \\
\frac{d \sigma_{E}}{d E_{r}} & \approx d_{E}^{2} \frac{\alpha Z^{2}}{E_{r} v^{2}} F_{E}^{2} .
\end{aligned}
$$

Note the $1 / E_{r}$ dependence of these differential cross sections, stemming from the propagator of the (massless) photon. As compared to a standard WIMP case, where the particle exchanged with the nucleon has typically an electroweak scale mass, this will largely boost the number of events in direct detection events, since the recoil energy considered in these experiments is typically of order $5-50 \mathrm{keV}$ (see below). This explains why below the constraints from direct detection through dipoles will be competitive, despite the fact that they involve loop suppressed quantities.

In eqs. (5.5)-(5.6), $\alpha=1 / 137, Z$ is the atomic number, $F_{E}$ and $F_{M}$ are two nuclear form factors, and $G$ is a dimensionless quantity depending on the nuclear spin $J$ and the nuclear magnetic dipole $d_{N}[17]$ :

$$
G=\frac{2(J+1)}{3 J}\left(\frac{d_{N} A}{d_{n}}\right)^{2} \approx 7256.78(\text { for } \mathrm{Xe}),
$$

where $d_{n}=e /\left(2 m_{p}\right)$ is the nuclear magneton, and $A$ is the mass number. For the nuclear form factors, we adopt the following approximate expressions [17]:

$$
\begin{aligned}
& F_{E}=3\left[\frac{\sin (q r)-q r \cos (q r)}{(q r)^{3}}\right] e^{-q^{2} s^{2}}, \\
& F_{M}=\frac{\sin (q \tilde{r})}{q \tilde{r}} \Theta(q \tilde{r}<2.55)+0.21 \Theta(2.55<q \tilde{r}<4.5),
\end{aligned}
$$

where $r=1.12 A^{1 / 3} \mathrm{fm}, \tilde{r}=A^{1 / 3} \mathrm{fm}, s=1 \mathrm{fm}$, and $q=\sqrt{2 E_{r} m_{N}}$. The $\Theta$ function takes either the value 1 or the value 0 , depending on whether the condition it involves is satisfied. For Xe targets, $q \tilde{r}<4.5$ corresponds to $E_{r}<117 \mathrm{keV}$, which in practice is always satisfied.

In the magnetic dipole cross section (5.5) we have included both SI $\left(\propto F_{E}^{2}\right)$ and SD $\left(\propto F_{M}^{2}\right)$ parts because they can be equally important. For example, when $m_{\chi}=m_{N}$ and $v=1.2 v_{\min }$, the ratio of the two parts at $E_{r}=30 \mathrm{keV}$ is about 1.6 . For the electric dipole cross section (5.6) we have neglected a possible SD contribution because it is highly suppressed. The fundamental reason for this is that electric charges of nucleons can be added coherently, unlike magnetic moments of nucleons. As a consequence, the electric dipole cross section is generally much larger than the magnetic one when $d_{M} \simeq d_{E}$.

For both eqs. (5.5) and (5.6), the velocity dependence can be written as follows:

$$
\frac{d \sigma}{d E_{r}}=\frac{1}{v^{2}}\left(\frac{d \sigma_{g}}{d E_{r}}+v^{2} \frac{d \sigma_{h}}{d E_{r}}\right)
$$

where $d \sigma_{g} / d E_{r}$ and $d \sigma_{h} / d E_{r}$ are velocity independent. Substituting eq. (5.10) into eq. (5.1), we obtain

$$
\frac{d R}{d E_{r}}=N_{T} n_{\chi} \epsilon\left(E_{r}\right)\left[\frac{d \sigma_{g}}{d E_{r}} g\left(v_{\min }\right)+\frac{d \sigma_{h}}{d E_{r}} h\left(v_{\min }\right)\right],
$$




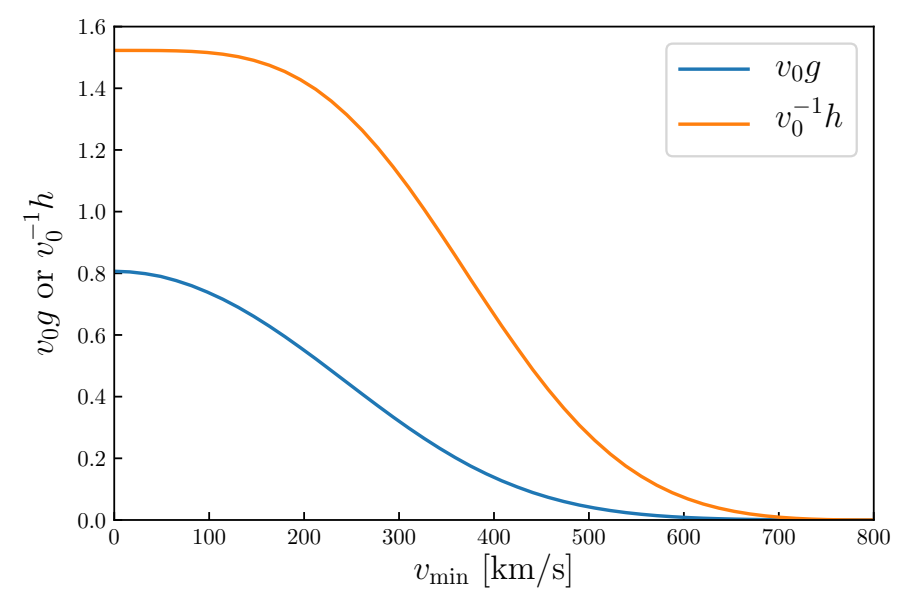

Figure 2. Results of $g\left(v_{\min }\right)$ and $h\left(v_{\min }\right)$ in eqs. (5.12) and (5.13).



Figure 3. Event rates for DM-nucleus scattering via magnetic and electric dipoles compared with the standard (contact-interaction) case. Shown examples take $d_{M}=5 \times 10^{-20} \mathrm{ecm}, d_{E}=1 \times 10^{-22}$ ecm, $\sigma_{n}=10^{-46} \mathrm{~cm}^{2}$, and $m_{\chi}=100 \mathrm{GeV}$. Dashed curves assume ideal detection efficiency and solid curves take the XENON-1T detection efficiency [10] into account.

where

$$
\begin{aligned}
& g\left(v_{\min }\right) \equiv \int v^{-1} f_{\oplus}(\mathbf{v}) \Theta\left(v-v_{\min }\right) d^{3} \mathbf{v}, \\
& h\left(v_{\min }\right) \equiv \int v f_{\oplus}(\mathbf{v}) \Theta\left(v-v_{\min }\right) d^{3} \mathbf{v},
\end{aligned}
$$

can be computed independently of the cross section and of the kinematics of DM-nucleus scattering. When numerically evaluating the integrals, we take eq. (5.3) with $\tilde{v}=\left(v^{2}+\right.$ $\left.v_{\oplus}^{2}-2 v v_{\oplus} \cos \theta\right)^{1 / 2}$ where $\theta$ is the angle between $\mathbf{v}_{\oplus}$ and $\mathbf{v}$, and integrate $\theta$ from 0 to $\pi, v$ from 0 to $v_{0}+v_{\text {esc }}$. The results are presented in figure 2 .

Using eq. (5.11), we plot in figure 3 the differential event rates (dashed curves) for $d_{M}=5 \times 10^{-20} \mathrm{ecm}, d_{E}=1 \times 10^{-22} \mathrm{ecm}$, assuming $m_{\chi}=100 \mathrm{GeV}$ and a $10^{3} \mathrm{~kg}$ liquid 
Xe target. For comparison, we also present a curve for the following SI contact-interacting cross section:

$$
\frac{d \sigma_{\text {contact }}}{d E_{r}}=\sigma_{n} \frac{m_{N} A^{2} F_{E}^{2}}{2 \mu_{\chi n}^{2} v^{2}}
$$

where $\mu_{\chi n}=m_{\chi} m_{n} /\left(m_{\chi}+m_{n}\right)$ is the DM-nucleon reduced mass. For the DM-nucleon cross section $\sigma_{n}$ we have taken the typical value that can be probed by direct detection experiment today, $\sigma_{n}=10^{-46} \mathrm{~cm}^{2}$. For the solid curves in figure 3 , we have included the detection efficiency of XENON-1T, which is taken from figure 1 in ref. [10]. The range of relevant recoil energy in direct detection experiments is relatively narrow, as below $\sim 5 \mathrm{keV}$ and above $\sim 50 \mathrm{keV}$ the efficiency is suppressed (compare dashed and solid lines in figure 3 ). As can be seen in this figure too, within this range, the dipole lines display, as expected, an extra $1 / E_{r}$ dependence with respect to the contact interaction case.

As a result of this relatively narrow range of recoil energy, it is possible to recast the XENON-1T bounds obtained for a contact interaction into bounds holding for the massless mediator $\left(\propto 1 / E_{r}\right)$ case of interest here. To this end, we apply a spectrumfitting technique previously adopted in ref. [60], namely using eq. (5.14) to fit the dipoleinteracting recoil spectra. Specifically, for a given set of $d_{M}\left(d_{E}\right)$ and $m_{\chi}$, one can correspondingly find values of $\sigma_{n}$ and $m_{\chi}^{\prime}$ (usually different from $m_{\chi}$ ) that minimizes the integral $\int\left(d R_{\text {dipole }} / d E_{r}-d R_{\text {contact }} / d E_{r}\right)^{2} d E_{r}$ where $d R_{\text {dipole }} / d E_{r}$ and $d R_{\text {contact }} / d E_{r}$ are the dipole- and contact-interacting spectra (including the detection efficiency). The minimization is performed under an additional constraint that their total rates are equal. We find that after the minimization, the two spectra are usually very close, with relative differences typically below $20 \%$, which is consistent with the conclusion in ref. [60]. By mapping $d_{M}\left(d_{E}\right)-m_{\chi}$ to $\sigma_{n}-m_{\chi}^{\prime}$ and taking the XENON-1T limit from ref. [10], we obtain the bounds on $d_{M}$ and $d_{E}$, presented in figure 4 . For comparison, we also show in figure 5 other known bounds on DM electromagnetic dipoles from indirect detection, CMB observations, and collider searches. These bounds in the WIMP regime are known to be much weaker than that from direct detection.

Figure 4 shows that the possibility that the axial operators would be responsible for the observed DM relic density is already excluded by direct detection experiments within the $6.8 \mathrm{GeV}<m_{\chi}<1.9 \mathrm{TeV}$ range for charged leptons. Future experiments such as XENON$\mathrm{nT}$ will enlarge this range significantly. For the vector case, although it is beyond the current best limit from XENON-1T, future XENON-nT will be able to probe the range $11.8 \mathrm{GeV}<m_{\chi}<205 \mathrm{GeV}$. The axial case is more constrained than the vector one because it requires a larger coefficient to account for the relic density constraint due to $p$-wave annihilation, see eq. (2.16) and table 1. For the $S, P$ and $T$ cases the additional $m_{f} / m_{\chi}$ dependence of the dipoles decreases the sensitivity for high values of $m_{\chi}$ but boosts it for low values. The sensitivity also splits among generations of fermions. Taking the tensor case in the right panel of figure 4 as an example, XENON-1T has excluded $m_{\chi} \lesssim 189 \mathrm{GeV}$ for $f=\mu$ while for $f=\tau$ this bound increases to $m_{\chi} \lesssim 1.2 \mathrm{TeV}$. The future experiment XENON-nT will be able to improve the mass bound by roughly a factor of three.

The four fermion interactions also induce fluxes of cosmic rays from the annihilation into charged fermion they induce today at tree level in the galactic center and dwarf 



Figure 4. The loop-induced magnetic dipole $d_{M}$ (left panel) and electric dipole $d_{E}$ compared with the XENON-1T [10] limit and the future XENON-nT [58] sensitivity. The theoretical expectations of $d_{M}$ and $d_{E}$ are computed according to eqs. (4.1)-(4.4). Dipoles for other fermions than the ones considered in these panels can be obtained for example from the $\tau$ case from a simple multiplication by $Q_{f} / Q_{\tau}$ for $V$ and $A$ and by $Q_{f} m_{f} /\left(Q_{\tau} m_{\tau}\right)$ for $S, P$ and $T$.



Figure 5. Comparison of direct detection bounds with other known bounds on DM electric/magnetic dipoles. The direct detection bounds (XENON-1T) are the same as those in figure 4 . The CMB bound is taken from ref. [21]. The indirect detection bounds, taken from ref. [59], are derived from FERMI-LAT constraints on $\gamma$-rays from the Galaxy (labelled as FERMI-Galaxy) and dwarf Spheroidal galaxies (FERMI-dSphs). The collider bound is obtained from LHC mono-jet searches [32].

galaxies. Indirect detection experiments give upper bounds on these fluxes which are generally translated into upper bounds on the annihilation cross section assuming $\Omega_{\chi} h^{2}=$ 0.12 (i.e. not looking at the implications that this annihilation cross section could have on the relic density). In figure 6 we show for the $V$ case how these bounds compare with the bounds that can be obtained on the same cross sections from the bound that direct detection set on the dipoles and thus on the coefficient of the four-fermion operators (assuming $\Omega_{\chi} h^{2}=0.12$ anyway there too). As figure 6 shows, despite that at tree level direct detection experiments are not much sensitive to the four-fermion operators for charged leptons or 

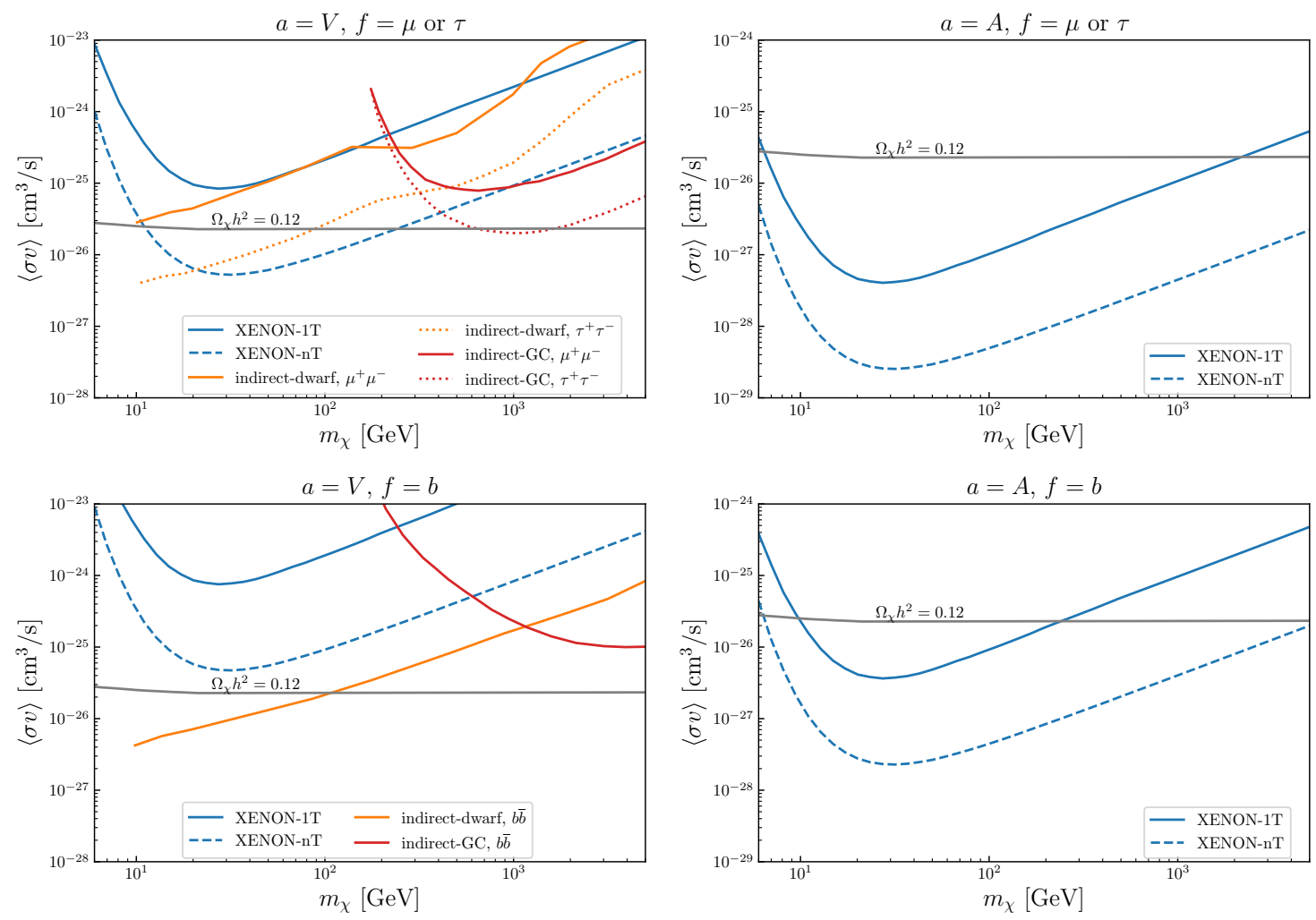

Figure 6. Direct detection bounds on $\langle\sigma v\rangle$ in comparison with indirect detection bounds, both obtained assuming $\Omega_{\chi} h^{2}=0.12$. For direct detection, we take the XENON-1T/nT bounds on $d_{M}$ obtained in figure 4 and recast them to bounds on $\epsilon$ 's according to table 2, and further to bounds on $\langle\sigma v\rangle$ according to table 1, assuming $a=V / A$. For indirect detections, the "indirect-dwarf" bounds are taken from ref. [61], and "indirect-GC" from [62], assuming that DM annihilates to $\tau^{+} \tau^{-}, \mu^{+} \mu^{-}$ or $b \bar{b}$. The horizontal line shows the thermal cross section value.

heavy quarks, when the loop-induced dipoles are taken into account, direct detection offers competitive constraints on such operators in comparison with indirect detection. For the $A$ case (and similarly for the $S$ case) indirect detection constraints are known to be weak as in this case the annihilation is of the $p$-wave type. But the bounds from dipole induced direct detection remains fully relevant, as given in figure 6 too.

Explicit UV complete models can be constrained according to the combination of effective operators they lead to. For the model considered in section 3.4, which in a characteristic way gives both $V$ and $A$ interactions with similar weights, we show in figure 7 upper bounds on the Yukawa coupling $y$ of eq. (3.15), using the dipoles obtained in eq. (3.19) (EFT) and eq. (3.22) (UV). As previously discussed in section 3.4, the difference between the two approaches shown in figure 7 is small. The current XENON-1T limit excludes the mass range $8.1 \mathrm{GeV} \lesssim m_{\chi} \lesssim 94 \mathrm{GeV}$ while future XENON-nT will be able to probe the $6.2 \mathrm{GeV} \lesssim m_{\chi} \lesssim 843 \mathrm{GeV}$ range.

As mentioned in the introduction, ref. [16] also studied the direct detection signals that could be induced by four-fermion effective operators (involving two charged leptons) via 




Figure 7. Constraints on the UV complete model defined in eq. (3.15) for any charged lepton $f$ and $m_{\phi}=2 m_{\chi}$. EFT/UV indicates that the curve takes the loop-induced dipole in eq. (3.19)/(3.22).

DM-photon interactions induced at the one-loop level. This calculation was done by calculating the loop directly from the 4 -fermion operator. This corresponds essentially to what we did in section 3.1, i.e. to what we get for the $s$-channel case. ${ }^{10}$ The two-step calculation we did, calculating first the various moments that are induced and subsequently computing what it gives for direct detection (instead of calculating directly the direct detection cross section) is useful as it identifies for each case what kind of electromagnetic interactions is induced (with what it implies for each of these interactions). Phenomenologically figure 4 also shows that the dramatic improvements of direct detection experiments in the last decade imply that the cases with chirality-flip suppressions (i.e. the $S, P$ and $T$ cases) are also testable (see e.g. the scalar case for $f=\tau$ in figure 4). As also shown above, electromagnetic interactions induced at the one-loop level are relevant not only for charged leptons but also for the heavy quark case. ${ }^{11}$

\section{Conclusion}

In the presence of four-fermion effective interactions of dark matter (DM) with Standard Model (SM) fermions, electromagnetic dipoles of DM can easily be generated, due to the loop process illustrated in figure 1 . This is the case in particular if the operators are generated through the exchange of a $t$-channel mediator. We study systematically for all possible effective interactions the loop-induced dipoles and find that, if they are not identically vanishing, the electromagnetic dipoles in the WIMP paradigm are typically of the order of $10^{-21}\left(10^{-20}\right)$ ecm for vector (axial-vector) interactions, or of $10^{-20} m_{f} / m_{\chi}$ ecm for scalar, pseudo-scalar, and tensor interactions, see eqs. (4.1)-(4.4). Calculations for a UV complete model give very similar results.

\footnotetext{
${ }^{10}$ At one loop one is left therefore with a vanishing contribution for the $S, P$ and $A$ operators and a suppressed charge radius contribution for the $\mathrm{V}$ operator.

${ }^{11}$ For this case, it would be interesting to compute the loop-level direct detection signals that are induced via gluon exchange rather than photon exchange, which is beyond the scope of this work.
} 
Via photon exchange such values imply observable nuclear recoil signals in direct detection experiments. This provides (or will provide) the most stringent constraints for various operators, in particular for axial or scalar operators, as well as for operators involving for instance muons. So far XENON-1T has excluded the loop-induced electromagnetic dipoles for some types of effective interactions in certain mass ranges - see figure 4 . Future multiton liquid xenon experiments with substantially improved sensitivity will be able to probe the dipoles for all types of effective interactions over much broader mass ranges.

\section{Acknowledgments}

We thank Xiaoyong Chu and Laurent Vanderheyden for useful discussions. This work is supported by the "Probing dark matter with neutrinos" ULB-ARC convention and by the F.R.S./FNRS under the Excellence of Science (EoS) project No. 30820817 - be.h "The $H$ boson gateway to physics beyond the Standard Model".

\section{A Loop calculations}

In this appendix, we present the details of our loop calculations. Starting from eq. (3.4), we first compute the traces:

$$
\begin{aligned}
& \operatorname{tr}\left[\frac{1}{k_{2}-m_{f}} \gamma^{\mu} \frac{1}{\not k_{1}-m_{f}} P_{L}\right]=2 m_{f}\left(k_{1}^{\mu}+k_{2}^{\mu}\right) D_{k}, \\
& \operatorname{tr}\left[\frac{1}{\not k_{2}-m_{f}} \gamma^{\mu} \frac{1}{\not k_{1}-m_{f}} \gamma^{\nu} P_{L}\right]=2\left[k_{1}^{\mu} k_{2}^{\nu}+k_{2}^{\mu} k_{1}^{\nu}+\left(m_{f}^{2}-k_{2} \cdot k_{1}\right) g^{\mu \nu}-i \epsilon^{\mu \nu \rho \lambda} k_{1 \rho} k_{2 \lambda}\right] D_{k}, \\
& \operatorname{tr}\left[\frac{1}{\not k_{2}-m_{f}} \gamma^{\mu} \frac{1}{\not k_{1}-m_{f}} \sigma^{\rho \lambda} P_{L}\right]=2 i m_{f}\left[\left(k_{1}^{\rho}-k_{2}^{\rho}\right) g^{\lambda \mu}-\lambda \leftrightarrow \rho\right]+2 m_{f} \epsilon^{\lambda \mu \rho \nu}\left(k_{1}-k_{2}\right)_{\nu} D_{k},
\end{aligned}
$$

where

$$
D_{k} \equiv \frac{1}{k_{2}^{2}-m_{f}^{2}} \frac{1}{k_{1}^{2}-m_{f}^{2}} .
$$

If $P_{L}$ in the above traces is replaced by $P_{R}$, the results are similar except that $\epsilon^{\mu \nu \rho \lambda}$ and $\epsilon^{\lambda \mu \rho \nu}$ flip their signs.

Next, we plug the traces into the loop integral and integrate out $k$, assuming the mass hierarchy:

$$
m_{\phi} \gg m_{\chi} \gg m_{f}
$$

Taking the case of eq. (A.1) with $G_{X}^{(t)}$ in eq. (3.7) for example, we have:

$$
\int \frac{d^{4} k}{(2 \pi)^{4}} \operatorname{tr}\left[\frac{1}{\not k_{2}-m_{f}} \gamma^{\mu} \frac{1}{\not k_{1}-m_{f}} P_{L}\right] \frac{1}{k^{2}-m_{\phi}^{2}} \approx-\frac{i}{16 \pi^{2}}\left(p_{1}+p_{2}\right)^{\mu} \frac{m_{f}}{m_{\phi}^{2}} .
$$

The loop integral is computed using Package-X [63] and expanded in $q^{2}, m_{f}$, and $m_{\chi}$. Only the leading order term is taken. Note that the integral is free from UV divergences 
because for $k \rightarrow \infty$ the integral behaves like $\int k^{-5} d^{4} k$ where the trace accounts for $k^{-3}$ according to eq. (A.1) and the remaining part accounts for $k^{-2}$.

With the result in eq. (A.6) and $1 / m_{\phi}^{2}=\epsilon_{a}^{L} G_{F}$, we see that $\mathcal{F}^{\mu}$ introduced in eq. (3.1) for $a=S$ and $P$ should be

$$
\mathcal{F}^{\mu}=\Gamma^{a} e Q_{f} \frac{\epsilon_{a}^{L} G_{F} m_{f}}{16 \pi^{2}}\left(p_{1}+p_{2}\right)^{\mu} .
$$

Then for $\overline{u_{2}} \mathcal{F}^{\mu} u_{1}$, it can be decomposed into the form factors in eq. (3.2) using the following identities:

$$
\begin{aligned}
\overline{u_{2}}\left(p_{1}+p_{2}\right)^{\mu} u_{1} & =\overline{u_{2}}\left[i \sigma^{\mu \nu} q_{\nu}\right] u_{1}+2 m_{\chi} \overline{u_{2}} \gamma^{\mu} u_{1}, \\
\overline{u_{2}}\left(p_{1}+p_{2}\right)^{\mu} i \gamma^{5} u_{1} & =\overline{u_{2}}\left[-\sigma^{\mu \nu} \gamma^{5} q_{\nu}\right] u_{1},
\end{aligned}
$$

where $q \equiv p_{1}-p_{2}$. Eq. (A.8) is the well-known Gordon identity (due to the definition of $q$, our convention differs from that in ref. ([64] page 186) by a minus sign of $q$ ). Eq. (A.9) is similar but with additional $\gamma^{5}$. It can be derived as follows:

$$
\begin{aligned}
\overline{u_{2}}\left[i \sigma^{\mu \nu} \gamma^{5} q_{\nu}\right] u_{1} & =-\frac{1}{2} \overline{u_{2}}\left[\gamma^{\mu}, \gamma^{\nu}\right] \gamma^{5} q_{\nu} u_{1} \\
& =-\frac{1}{2} \overline{u_{2}}\left[\gamma^{\mu} \not p_{1} \gamma^{5}-\gamma^{\mu} \not p_{2} \gamma^{5}-\not p_{1} \gamma^{\mu} \gamma^{5}+\not p_{2} \gamma^{\mu} \gamma^{5}\right] u_{1} \\
& =\frac{1}{2} \overline{u_{2}}\left[\left(2 p_{2}^{\mu}-\not p_{2} \gamma^{\mu}\right) \gamma^{5}+\left(2 p_{1}^{\mu}-\gamma^{\mu} \not p_{1}\right) \gamma^{5}\right] u_{1} \\
& =\left(p_{1}+p_{2}\right)^{\mu} \overline{u_{2}} \gamma^{5} u_{1},
\end{aligned}
$$

where in the second row the first and last terms cancel out because $\not p_{1} \gamma^{5} u_{1}=-m_{\chi} \gamma^{5} u_{1}$ and $\overline{u_{2}} \not p_{2}=\overline{u_{2}} m_{\chi}$, and in the third row the $\not p_{2} \gamma^{\mu}$ and $\gamma^{\mu} \not p_{1}$ terms cancel out for the same reason.

According to eqs. (A.8) and (A.9), eq. (A.7) generates magnetic and electric dipoles for $a=S$ and $P$, respectively. The values are already listed in table 2. For other cases, the calculations are similar: we plug eq. (A.2) or (A.3) into the loop integral to obtain $\mathcal{F}^{\mu}$ and use eq. (A.8) or (A.9) to extract the dipole form factors. In general, when the resulting $\mathcal{F}^{\mu}$ contains $\not p_{1}$ and $\not p_{2}$, after applying the Dirac algebra and on-shell conditions $\left(\not p_{1} u_{1}=m_{\chi} u_{1}\right.$ and $\left.\overline{u_{2}} \phi_{2}=\overline{u_{2}} m_{\chi}\right)$, they can be converted to linear combinations of $\left(p_{1}+p_{2}\right)^{\mu}$ and $\left(p_{1}-p_{2}\right)^{\mu}$. Terms containing the latter cancel out or can be neglected due to the Ward identity. In Package-X [63], the dipole form factors can be extracted using dedicated projectors, and we have verified that this approach leads to the same results.

As for the UV complete example introduced in section 3.4, there are two diagrams contributing to the magnetic dipole: one with the photon coupled to the charge fermion $f$ and the other with photon coupled to the charged scalar $\phi$. We refer to the former and the latter as diagrams (i) and (ii), respectively. Their amplitudes read

$$
\begin{aligned}
i \mathcal{M}_{(\mathrm{i})} & =i \int \frac{d^{4} k}{(2 \pi)^{4}} \overline{u_{2}} P_{R} \frac{1}{\not k_{2}-m_{f}} i e Q_{f} \gamma^{\mu} \frac{1}{\not k_{1}-m_{f}} P_{L} u_{1} \varepsilon_{\mu} \frac{g_{R} g_{R}^{*}}{k^{2}-m_{\phi}^{2}}, \\
i \mathcal{M}_{(\mathrm{ii})} & =i \int \frac{d^{4} k}{(2 \pi)^{4}} \overline{u_{2}} P_{R} \frac{1}{\not k-m_{f}} P_{L} u_{1} \varepsilon_{\mu} i e Q_{\phi}\left(k_{1}+k_{2}\right)^{\mu} \frac{1}{k_{1}^{2}-m_{\phi}^{2}} \frac{1}{k_{2}^{2}-m_{\phi}^{2}} g_{R} g_{R}^{*},
\end{aligned}
$$


where $Q_{\phi}$ is the electric charge of $\phi$. After integrating out the loop momentum, we find

$$
\begin{aligned}
\mathcal{M}_{(\mathrm{i})}= & \frac{e\left|g_{R}\right|^{2} Q_{f}}{32 \pi^{2}} \overline{u_{2}} \gamma^{\mu} u_{1} \varepsilon_{\mu}\left[\frac{1}{2 \epsilon}+\frac{1}{4}+\log \left(\frac{\mu}{m_{\phi}}\right)\right] \\
& +\frac{e\left|g_{R}\right|^{2} Q_{f}}{32 \pi^{2} m_{\phi}^{2}} \overline{u_{2}} \gamma^{\mu} u_{1} \varepsilon_{\mu}\left[\frac{1}{2} m_{\chi}^{2}-\frac{1}{2} m_{f}^{2}\right] \\
& +\frac{e\left|g_{R}\right|^{2} Q_{f}}{32 \pi^{2} m_{\phi}^{2}} \overline{u_{2}} i \sigma^{\mu \nu} q_{\nu} u_{1} \varepsilon_{\mu}\left[-\frac{1}{3} m_{\chi}\right] \\
& +\left[\overline{u_{2}} \gamma^{\mu} \gamma^{5} u_{1} \text { terms }\right], \\
\mathcal{M}_{(\mathrm{ii})}= & \frac{e\left|g_{R}\right|^{2} Q_{\phi}}{32 \pi^{2}} \overline{u_{2}} \gamma^{\mu} u_{1} \varepsilon_{\mu}\left[\frac{1}{2 \epsilon}+\frac{1}{4}+\log \left(\frac{\mu}{m_{\phi}}\right)\right] \\
& +\frac{e\left|g_{R}\right|^{2} Q_{\phi}}{32 \pi^{2} m_{\phi}^{2}} \overline{u_{2}} \gamma^{\mu} u_{1} \varepsilon_{\mu}\left[\frac{1}{2} m_{\chi}^{2}-\frac{1}{2} m_{f}^{2}\right] \\
& +\frac{e\left|g_{R}\right|^{2} Q_{\phi}}{32 \pi^{2} m_{\phi}^{2}} \overline{u_{2}} i \sigma^{\mu \nu} q_{\nu} u_{1} \varepsilon_{\mu}\left[+\frac{1}{6} m_{\chi}\right] \\
& +\left[\overline{u_{2}} \gamma^{\mu} \gamma^{5} u_{1} \text { terms }\right] .
\end{aligned}
$$

Here $\overline{u_{2}} \gamma^{\mu} \gamma^{5} u_{1}$ terms are not important because they cancel out in the final result, as we have verified explicitly in the calculation.

As is manifest, the UV divergences, as well as all finite $\overline{u_{2}} \gamma^{\mu} u_{1}$ contributions of eqs. (A.12) and (A.13) cancel out when $Q_{\phi}+Q_{f}=0$, which is required by the charge conservation of eq. (3.15).

After all the cancellations, only the magnetic dipole terms exist. Comparing the last row of eq. (A.12) to eq. (3.19), we see that $\mathcal{M}_{(\mathrm{i})}$ reproduces the dipole obtained in the simplified approach. When the full theory is taken into account, the additional contribution due to $\mathcal{M}_{\text {(ii) }}$ is roughly half the size of the previous one, assuming $m_{f} \ll m_{\chi}$. Taking $Q_{\phi}=-Q_{f}$ and summing the two diagrams together, we obtain the result in eq. (3.22).

Open Access. This article is distributed under the terms of the Creative Commons Attribution License (CC-BY 4.0), which permits any use, distribution and reproduction in any medium, provided the original author(s) and source are credited.

\section{References}

[1] G. Jungman, M. Kamionkowski and K. Griest, Supersymmetric dark matter, Phys. Rept. 267 (1996) 195 [hep-ph/9506380] [INSPIRE].

[2] G. Bertone, D. Hooper and J. Silk, Particle dark matter: Evidence, candidates and constraints, Phys. Rept. 405 (2005) 279 [hep-ph/0404175] [INSPIRE].

[3] J.L. Feng, Dark Matter Candidates from Particle Physics and Methods of Detection, Ann. Rev. Astron. Astrophys. 48 (2010) 495 [arXiv: 1003.0904] [InSPIRE].

[4] G. Arcadi et al., The waning of the WIMP? A review of models, searches, and constraints, Eur. Phys. J. C 78 (2018) 203 [arXiv: 1703.07364] [INSPIRE]. 
[5] L. Roszkowski, E.M. Sessolo and S. Trojanowski, WIMP dark matter candidates and searches - current status and future prospects, Rept. Prog. Phys. 81 (2018) 066201 [arXiv: 1707.06277] [INSPIRE].

[6] M. Schumann, Direct Detection of WIMP Dark Matter: Concepts and Status, J. Phys. G 46 (2019) 103003 [arXiv:1903.03026] [INSPIRE].

[7] G. Busoni, A. De Simone, E. Morgante and A. Riotto, On the Validity of the Effective Field Theory for Dark Matter Searches at the LHC, Phys. Lett. B 728 (2014) 412 [arXiv: 1307.2253] [INSPIRE].

[8] G. Busoni, A. De Simone, J. Gramling, E. Morgante and A. Riotto, On the Validity of the Effective Field Theory for Dark Matter Searches at the LHC, Part II: Complete Analysis for the s-channel, JCAP 06 (2014) 060 [arXiv:1402.1275] [INSPIRE].

[9] G. Busoni, A. De Simone, T. Jacques, E. Morgante and A. Riotto, On the Validity of the Effective Field Theory for Dark Matter Searches at the LHC Part III: Analysis for the t-channel, JCAP 09 (2014) 022 [arXiv:1405.3101] [INSPIRE].

[10] XENON collaboration, Dark Matter Search Results from a One Ton-Year Exposure of XENON1T, Phys. Rev. Lett. 121 (2018) 111302 [arXiv:1805.12562] [INSPIRE].

[11] LUX collaboration, Results from a search for dark matter in the complete LUX exposure, Phys. Rev. Lett. 118 (2017) 021303 [arXiv: 1608. 07648] [INSPIRE].

[12] PandaX-II collaboration, Dark Matter Results From 54-Ton-Day Exposure of PandaX-II Experiment, Phys. Rev. Lett. 119 (2017) 181302 [arXiv:1708.06917] [INSPIRE].

[13] M. Pospelov and T. ter Veldhuis, Direct and indirect limits on the electromagnetic form-factors of WIMPs, Phys. Lett. B 480 (2000) 181 [hep-ph/0003010] [InSPIRE].

[14] K. Sigurdson, M. Doran, A. Kurylov, R.R. Caldwell and M. Kamionkowski, Dark-matter electric and magnetic dipole moments, Phys. Rev. D 70 (2004) 083501 [Erratum ibid. 73 (2006) 089903] [astro-ph/0406355] [INSPIRE].

[15] E. Masso, S. Mohanty and S. Rao, Dipolar Dark Matter, Phys. Rev. D 80 (2009) 036009 [arXiv: 0906.1979] [INSPIRE].

[16] J. Kopp, V. Niro, T. Schwetz and J. Zupan, DAMA/LIBRA and leptonically interacting Dark Matter, Phys. Rev. D 80 (2009) 083502 [arXiv:0907.3159] [InSPIRE].

[17] T. Banks, J.-F. Fortin and S. Thomas, Direct Detection of Dark Matter Electromagnetic Dipole Moments, arXiv:1007.5515 [INSPIRE].

[18] A.L. Fitzpatrick and K.M. Zurek, Dark Moments and the DAMA-CoGeNT Puzzle, Phys. Rev. D 82 (2010) 075004 [arXiv: 1007.5325] [INSPIRE].

[19] V. Barger, W.-Y. Keung and D. Marfatia, Electromagnetic properties of dark matter: Dipole moments and charge form factor, Phys. Lett. B 696 (2011) 74 [arXiv:1007.4345] [INSPIRE].

[20] J.-F. Fortin and T.M.P. Tait, Collider Constraints on Dipole-Interacting Dark Matter, Phys. Rev. D 85 (2012) 063506 [arXiv:1103.3289] [INSPIRE].

[21] E. Del Nobile, C. Kouvaris, P. Panci, F. Sannino and J. Virkajarvi, Light Magnetic Dark Matter in Direct Detection Searches, JCAP 08 (2012) 010 [arXiv: 1203.6652] [INSPIRE].

[22] N. Weiner and I. Yavin, How Dark Are Majorana WIMPs? Signals from MiDM and Rayleigh Dark Matter, Phys. Rev. D 86 (2012) 075021 [arXiv:1206. 2910] [InSPIRE]. 
[23] C.M. Ho and R.J. Scherrer, Anapole Dark Matter, Phys. Lett. B 722 (2013) 341 [arXiv: 1211.0503] [INSPIRE].

[24] M.I. Gresham and K.M. Zurek, Light Dark Matter Anomalies After LUX, Phys. Rev. D 89 (2014) 016017 [arXiv:1311.2082] [INSPIRE].

[25] Y. Gao, C.M. Ho and R.J. Scherrer, Anapole Dark Matter at the LHC, Phys. Rev. D 89 (2014) 045006 [arXiv:1311.5630] [INSPIRE].

[26] E. Del Nobile, G. Gelmini, P. Gondolo and J.-H. Huh, Generalized Halo Independent Comparison of Direct Dark Matter Detection Data, JCAP 10 (2013) 048 [arXiv: 1306. 5273] [INSPIRE].

[27] E. Del Nobile, G.B. Gelmini, P. Gondolo and J.-H. Huh, Direct detection of Light Anapole and Magnetic Dipole DM, JCAP 06 (2014) 002 [arXiv:1401.4508] [INSPIRE].

[28] J. Kopp, L. Michaels and J. Smirnov, Loopy Constraints on Leptophilic Dark Matter and Internal Bremsstrahlung, JCAP 04 (2014) 022 [arXiv: 1401.6457] [INSPIRE].

[29] X. Chu, J. Pradler and L. Semmelrock, Light dark states with electromagnetic form factors, Phys. Rev. D 99 (2019) 015040 [arXiv:1811.04095] [InSPIRE].

[30] J.H. Chang, R. Essig and A. Reinert, Light(ly)-coupled Dark Matter in the keV Range: Freeze-In and Constraints, JHEP 03 (2021) 141 [arXiv: 1911.03389] [INSPIRE].

[31] X. Chu, J.-L. Kuo and J. Pradler, Dark sector-photon interactions in proton-beam experiments, Phys. Rev. D 101 (2020) 075035 [arXiv: 2001.06042] [INSPIRE].

[32] C. Arina, A. Cheek, K. Mimasu and L. Pagani, Light and Darkness: consistently coupling dark matter to photons via effective operators, Eur. Phys. J. C 81 (2021) 223 [arXiv: 2005.12789] [INSPIRE].

[33] Y. Ali-Haïmoud, Testing dark matter interactions with CMB spectral distortions, Phys. Rev. D 103 (2021) 043541 [arXiv:2101.04070] [INSPIRE].

[34] J. Goodman, M. Ibe, A. Rajaraman, W. Shepherd, T.M.P. Tait and H.-B. Yu, Constraints on Dark Matter from Colliders, Phys. Rev. D 82 (2010) 116010 [arXiv:1008.1783] [INSPIRE].

[35] J. Brod, A. Gootjes-Dreesbach, M. Tammaro and J. Zupan, Effective Field Theory for Dark Matter Direct Detection up to Dimension Seven, JHEP 10 (2018) 065 [arXiv:1710.10218] [INSPIRE].

[36] F. Bishara, J. Brod, B. Grinstein and J. Zupan, DirectDM: a tool for dark matter direct detection, arXiv:1708.02678 [INSPIRE].

[37] F. Bishara, J. Brod, B. Grinstein and J. Zupan, Chiral Effective Theory of Dark Matter Direct Detection, JCAP 02 (2017) 009 [arXiv: 1611.00368] [INSPIRE].

[38] F. Bishara, J. Brod, B. Grinstein and J. Zupan, From quarks to nucleons in dark matter direct detection, JHEP 11 (2017) 059 [arXiv:1707.06998] [INSPIRE].

[39] M. Lindner, W. Rodejohann and X.-J. Xu, Coherent Neutrino-Nucleus Scattering and new Neutrino Interactions, JHEP 03 (2017) 097 [arXiv: 1612.04150] [INSPIRE].

[40] W. Rodejohann, X.-J. Xu and C.E. Yaguna, Distinguishing between Dirac and Majorana neutrinos in the presence of general interactions, JHEP 05 (2017) 024 [arXiv:1702.05721] [INSPIRE].

[41] M. Bauer and T. Plehn, Yet Another Introduction to Dark Matter: The Particle Physics Approach, Lect. Notes Phys. 959 (2019) 1. 
[42] E.W. Kolb and M.S. Turner, The Early Universe, Addison-Wesley Publishing, Boston U.S.A. (1990).

[43] P. Gondolo and G. Gelmini, Cosmic abundances of stable particles: Improved analysis, Nucl. Phys. B 360 (1991) 145 [INSPIRE].

[44] Particle Data Group collaboration, Review of Particle Physics, PTEP 2020 (2020) 083C01 [INSPIRE].

[45] M. Nowakowski, E.A. Paschos and J.M. Rodriguez, All electromagnetic form-factors, Eur. J. Phys. 26 (2005) 545 [physics/0402058] [INSPIRE].

[46] C. Giunti and A. Studenikin, Neutrino electromagnetic interactions: a window to new physics, Rev. Mod. Phys. 87 (2015) 531 [arXiv: 1403.6344] [INSPIRE].

[47] X.-J. Xu, Tensor and scalar interactions of neutrinos may lead to observable neutrino magnetic moments, Phys. Rev. D 99 (2019) 075003 [arXiv:1901.00482] [INSPIRE].

[48] J. Alwall, P. Schuster and N. Toro, Simplified Models for a First Characterization of New Physics at the LHC, Phys. Rev. D 79 (2009) 075020 [arXiv: 0810.3921] [InSPIRE].

[49] LHC New Physics Working Group collaboration, Simplified Models for LHC New Physics Searches, J. Phys. G 39 (2012) 105005 [arXiv:1105.2838] [inSPIRE].

[50] M.R. Buckley, D. Feld and D. Goncalves, Scalar Simplified Models for Dark Matter, Phys. Rev. D 91 (2015) 015017 [arXiv: 1410.6497] [INSPIRE].

[51] J. Abdallah et al., Simplified Models for Dark Matter and Missing Energy Searches at the $L H C$, arXiv:1409.2893 [INSPIRE].

[52] J. Abdallah et al., Simplified Models for Dark Matter Searches at the LHC, Phys. Dark Univ. 9-10 (2015) 8 [arXiv: 1506.03116] [INSPIRE].

[53] D. Abercrombie et al., Dark Matter benchmark models for early LHC Run-2 Searches: Report of the ATLAS/CMS Dark Matter Forum, Phys. Dark Univ. 27 (2020) 100371 [arXiv: 1507.00966] [INSPIRE].

[54] N.F. Bell, Y. Cai and R.K. Leane, Mono-W Dark Matter Signals at the LHC: Simplified Model Analysis, JCAP 01 (2016) 051 [arXiv: 1512.00476] [INSPIRE].

[55] C. Giunti and C.W. Kim, Fundamentals of Neutrino Physics and Astrophysics, Oxford University Press, Oxford U.K. (2007).

[56] T. Lin, Dark matter models and direct detection, PoS 333 (2019) 009 [arXiv:1904.07915] [INSPIRE].

[57] J.I. Read, The Local Dark Matter Density, J. Phys. G 41 (2014) 063101 [arXiv:1404.1938] [INSPIRE].

[58] XENON collaboration, Projected WIMP sensitivity of the XENONnT dark matter experiment, JCAP 11 (2020) 031 [arXiv:2007.08796] [INSPIRE].

[59] B.J. Kavanagh, P. Panci and R. Ziegler, Faint Light from Dark Matter: Classifying and Constraining Dark Matter-Photon Effective Operators, JHEP 04 (2019) 089 [arXiv: 1810.00033] [INSPIRE].

[60] T. Hambye, M.H.G. Tytgat, J. Vandecasteele and L. Vanderheyden, Dark matter direct detection is testing freeze-in, Phys. Rev. D 98 (2018) 075017 [arXiv:1807.05022] [InSPIRE]. 
[61] MAGIC, Fermi-LAT collaboration, Limits to Dark Matter Annihilation Cross-Section from a Combined Analysis of MAGIC and Fermi-LAT Observations of Dwarf Satellite Galaxies, JCAP 02 (2016) 039 [arXiv:1601.06590] [INSPIRE].

[62] H.E.S.S. collaboration, Search for dark matter annihilations towards the inner Galactic halo from 10 years of observations with H.E.S.S, Phys. Rev. Lett. 117 (2016) 111301 [arXiv: 1607.08142] [INSPIRE].

[63] H.H. Patel, Package-X: A Mathematica package for the analytic calculation of one-loop integrals, Comput. Phys. Commun. 197 (2015) 276 [arXiv:1503.01469] [INSPIRE].

[64] M. Peskin and D. Schroeder, An Introduction to quantum field theory, Addison-Wesley, Boston U.S.A. (1995). 